\title{
OPEN Spin and pseudospin solutions to Dirac equation and its thermodynamic properties using hyperbolic Hulthen plus hyperbolic exponential inversely quadratic potential
}

\author{
Ituen B. Okon ${ }^{1 凶}$, E. Omugbe ${ }^{2}$, Akaninyene D. Antia ${ }^{1}$, C. A. Onate ${ }^{3}$, Louis E. Akpabio ${ }^{1}$ \&
} O. E. Osafile 2

In this research article, the modified approximation to the centrifugal barrier term is applied to solve an approximate bound state solutions of Dirac equation for spin and pseudospin symmetries with hyperbolic Hulthen plus hyperbolic exponential inversely quadratic potential using parametric Nikiforov-Uvarov method. The energy eigen equation and the unnormalised wave function were presented in closed and compact form. The nonrelativistic energy equation was obtain by applying nonrelativistic limit to the relativistic spin energy eigen equation. Numerical bound state energies were obtained for both the spin symmetry, pseudospin symmetry and the non relativistic energy. The screen parameter in the potential affects the solutions of the spin symmetry and non-relativistic energy in the same manner but in a revised form for the pseudospin symmetry energy equation. In order to ascertain the accuracy of the work, the numerical results obtained was compared to research work of existing literature and the results were found to be in excellent agreement to the existing literature. The partition function and other thermodynamic properties were obtained using the compact form of the nonrelativistic energy equation. The proposed potential model reduces to Hulthen and exponential inversely quadratic potential as special cases. All numerical computations were carried out using Maple 10.0 version and Matlab 9.0 version softwares respectively.

Most of the quantum mechanical systems were investigated through bound state and scattering state solutions of relativistic and nonrelativistic wave equations with considerable potential models. Schrodinger wave equation constitutes the non-relativistic wave equation while Klein-Gordon, Dirac, Duffin-Kemmer-Petiau (DKP) constitute the relativistic wave equation ${ }^{1,2}$. The Dirac equation forms the foundation for the formulation of relativistic quantum mechanics ${ }^{3}$. Dirac equation has wide applications in physical sciences ranging from high energy physics, quantum information, quantum electrodynamics and quantum fluctuations ${ }^{4-7}$. The solutions of Dirac equation is applicable in describing the nuclear shell struture ${ }^{8-10}$. The concept of spin and pseudospin symmetries under Dirac formulation have been used extensively to explain the feature of deformed nuclei and super-deformation thereby establishing and effective shell model coupling scheme $\mathrm{e}^{11-13}$. Spin symmetry is use for the description of mesons. This arises when the difference of the potential between the repulsive Lorentz vector $V(r)$ and the attractive Lorentz scalar vector $S(r)$ is constant; that is, $\Delta=V(r)-S(r)$. However, pseudo symmetry arises when the sum of the potential of the repulsive Lorentz vector potential $V(r)$ and the attractive Lorentz scalar potential $S(r)$ is constant; that is $\sum(r)=V(r)+S(r)^{14-16}$. The Klein-Gordon equation as Lorentz scalar was use to describe spinless particle while Dirac equation was used for the description of spin-1/2 particles ${ }^{17-25}$. Meanwhile, the relativistic wave equation wether Dirac, Klein-Gordon, and DKP is consider either as mixed vector and scalar potentials or equal vector and scalar potentials ${ }^{26-30}$. In mathematical physics, the Dirac equation

${ }^{1}$ Theoretical Physics Group, Department of Physics, University of Uyo, Uyo, Nigeria. ${ }^{2}$ Department of Physics, Federal University of Petroleum Resources, Effurun, Nigeria. ${ }^{3}$ Department of Physical Sciences, Landmark University, Omu-Aran, Nigeria. ${ }^{\circledR}$ email: ituenokon@uniuyo.edu.ng 
with pseudo scalar potential can be handled as a Sturm-Liouville problem while the bound state spectrum of Dirac equation is relevant for the study of Zakharaov-Shabat eigenvalue problem which is applicable in the study of isospectral flow of soliton in plasma ${ }^{31-34}$. The solutions of Dirac equation with various considerable potentials within spin and pseudospin symmetries have been obtained by different authors using different methods such as: factorisation method ${ }^{35,36}$, asymptotic iteration $\operatorname{method~}^{37,38}$, Laplace transform technique ${ }^{39}$, Supersymmetric quantum mechanics approach ${ }^{40-42}$, path integral formulation ${ }^{43-45}$, Nikiforov-Uvarov method ${ }^{46,47}$ and others.

Both Dirac and Klein-Gordon equations have been studied with different potentials such as: Woods-Saxon, Rosen-Morse, Hulthen, Eckart, Manning Rosen plus Hellmann, Scarf potential and others ${ }^{48-57}$. The investigation of approximate bound state solutions of Dirac equation has been carried out by different authors. Okorie et al. ${ }^{58}$ investigated the solution of Dirac and Schrodinger equation with shifted Tietz-Wei potential where they obtained relativistic and nonrelativistic ro-vibrational energy spectra as well as numerical solutions for different diatomic molecules within the framework of factorisation method. Onyeaju et al. ${ }^{59}$ employed the Perkeris approximation to the centrifugal term to obtain approximate bound state solutions of Dirac equation with some thermodynamic properties for the deformed Hylleraas plus deformed Woods-Saxon potential. Similarly Ikhdair and Hamzavi ${ }^{60}$ studied Dirac bound state solutions of spherically ringed-shape q-deform Woods-Saxon potential for any l-state; where they obtained energy eigenvalue equation and the corresponding two components wave functions within the framework of Nikiforov-Uvarov method. Arda and Sever ${ }^{3}$ studied bound state solutions of Dirac equation for Kratzer potential with pseudo-scalar Coulomb term. Ortakaya et al. ${ }^{61}$ investigated bound state solutions of Dirac equation with Deng-Fan potential including a Coulomb tensor interaction within the framework of asymptotic iteration method where they presented their numerical results for spin and pseudospin limits. A lot of research work have been carried out by Ikot and co-authors on Dirac equation as seen in Refs ${ }^{62,63}$. Other research contributions on Dirac equations were presented in Refs ${ }^{64-66}$.

Some considerable potentials describing physical systems have been modelled as hyperbolic and trigonometric potentials because of their contributions in nuclear and high energy physics. Some authors have reported the applications and usefulness of such potential models. Ikhdair ${ }^{67}$ calculated a rotational and vibrational energies of diatomic molecules in Klein-Gordon equation using hyperbolic scalar and vector potential by means of parametric Nikiforov-Uvarov method. Ikot et al. ${ }^{68}$ studied Schrodinger equation with screen Kratzer potential in the presence of external magnetic and $\mathrm{AB}$ flux field using factorisation method. The eigenvalue and the eigenfunction of the system were presented in a close form while magnetisation and magnetic susceptibility were evaluated at zero and finite temperatures as well as thermodynamic properties. Dong et al. ${ }^{69}$ examined quantum information entropies for a squared tangent potential where the calculated position Shannon and momentum entropies that satisfies Beckner, Bialynicki and Mycieslki (BBM) inequality. Okon et al. ${ }^{70}$ obtained eigen solutions to Schrodinger equation with trigonometric Inversely quadratic plus Coulombic hyperbolic potential where they obtained energy eigen equation and normalised wave function using Nikiforov-Uvarov method. Onate ${ }^{71}$ examined bound state solutions of Schrodinger equation with second Poschl-Teller-like potential where he obtained vibrational partition function, mean energy and mean free energy where Poschl-Teller-like potential was expressed in the form of hyperbolic cosh and sinh. Okorie et al. ${ }^{72}$ obtained the ro-vibrational energy spectra of the improved deformed exponential-type potential using Greene-Aldrich approximation scheme and coordinate transformation where they obtained vibrational partition function and other thermodynamic properties using poisson summation formular.

In this research article, we solve bound state solutions of Dirac equation with equal scalar and vector potential using hyperbolic Hulthen plus hyperbolic Yukawa inversely quadratic potential under spin and pseudospin symmetries using the parametric Nikiforov-Uvarov method. This work is also extended to study some thermodynamic properties using the nonrelativistic energy eigen equation. This article is divided into seven sections. "Introduction" gives the brief introduction of the article. The basic theory of Dirac equation is presented in "Dirac equation for spin and pseudospin symmetry" section. Parametric Nikiforov-Uvarov method is presented in "The parametric Nikiforov-Uvarov (NU) method" section while the analytical solution of Dirac equation is presented in "Solution of Dirac equation with the proposed potential" section. The numerical results for both spin and pseudospin symmetries are presented in "Numerical results" section. Thermodynamic properties is presented in "Thermodynamic properties for the potential model" section while the discussion of numerical results and tables are done in "Discussion" section. The research article is concluded in "Conclusion" section.

\section{Dirac equation for spin and pseudospin symmetry}

The Dirac equation ${ }^{8}$ for spin-1/2 particles moving in a field of attractive scalar potential $S(r)$ and repulsive vector potential $V(r)$ is given as

$$
[\alpha \cdot p+\beta(M+S(r))] \psi(r)=[E-V(r)] \psi(r)
$$

where $E$ is the relativistic energy, $M$ is the mass of a single particle, $p=-i \Delta$ is the momentum operator, while $\alpha$ and $\beta$ are the $4 \times 4$ Pauli matrices defined as

$$
\alpha=\left(\begin{array}{cc}
0 & \sigma_{i} \\
\sigma_{i} & 0
\end{array}\right), \beta=\left(\begin{array}{cc}
I & 0 \\
0 & -I
\end{array}\right)
$$

Here $I$ is the $2 \times 2$ unitary matrix and $\sigma_{i}$ are the three vector spin matrices given as

$$
\sigma_{1}=\left(\begin{array}{ll}
0 & 1 \\
1 & 0
\end{array}\right), \sigma_{2}=\left(\begin{array}{cc}
0 & -i \\
i & 0
\end{array}\right), \sigma_{3}=\left(\begin{array}{ll}
1 & 0 \\
0 & 1
\end{array}\right)
$$


The total angular momentum $J$ and the spin orbit operator $\hat{K}=-\beta(\alpha \cdot L+1)$ of a particle commutes with the Dirac Hamiltonian in a central field where $L$ is the orbital angular momentum of the spherical nucleons. The eigenvalues of the spin-orbit coupling operator $\hat{K}$ are $\kappa=-\left(j+\frac{1}{2}\right)<0, j=\left(l-\frac{1}{2}\right)$ for unaligned spin and $\kappa=\left(j+\frac{1}{2}\right)>0, j=\left(l+\frac{1}{2}\right)$ for aligned spin respectively. Thus, the spinor wave function can be classified according to the angular momentum $j$, the spin orbit quantum number $\kappa$ and the radial quantum number $n$. The wave function can then be written as $\kappa$ and the radial quantum number $n$. The wave function can then be written as

$$
\psi(\vec{r})=\left(\begin{array}{c}
\xi(\vec{r}) \\
\phi(\vec{r})
\end{array}\right)=\frac{1}{r}\left(\begin{array}{c}
F_{n k}(r) Y_{j m}^{l}(\theta, \varphi) \\
i G_{n k}(r) Y_{j m}^{\tilde{l}}(\theta, \varphi)
\end{array}\right)
$$

where $F_{n k}$ and $G_{n k}$ are the upper and lower radial wave functions respectively. $Y_{j m}^{l}(\theta, \varphi)$ and $Y_{j m}^{\tilde{l}}(\theta, \varphi)$ are the spin and pseudospin spherical harmonics respectively. $m$ is the projection of angular momentum on the z-axis. Substituting Eqs. (1)-(4) and by making use of the following relations

$$
\begin{gathered}
(\hat{\sigma} \cdot \vec{A}) \cdot(\hat{\sigma} \cdot \vec{B})=\vec{A} \cdot \vec{B}+i \hat{\sigma} \cdot(\vec{A} \times \vec{B}) \\
\hat{\sigma} \cdot \hat{p}=\hat{\sigma} \cdot \hat{r}\left(\hat{r} \cdot \hat{p}+\frac{i \hat{\sigma} \tilde{l}}{r}\right)
\end{gathered}
$$

Together with the additional properties

$$
\left.\begin{array}{l}
(\hat{\sigma} \cdot \vec{L}) Y_{j m}^{\tilde{l}}(\theta, \varphi)=(\kappa-1) Y_{j m}^{\tilde{l}}(\theta, \varphi) \\
(\hat{\sigma} \cdot \vec{L}) Y_{j m}^{l}(\theta, \varphi)=(\kappa-1) Y_{j m}^{l}(\theta, \varphi) \\
(\hat{\sigma} \cdot \hat{r}) Y_{j m}^{\tilde{l}}(\theta, \varphi)=-Y_{j m}^{l}(\theta, \varphi) \\
(\hat{\sigma} \cdot \hat{r}) Y_{j m}^{l}(\theta, \varphi)=-Y_{j m}^{\tilde{l}}(\theta, \varphi)
\end{array}\right\}
$$

result into two coupled differential equations whose solutions constitutes the upper $F_{n k}$ and the lower $G_{n k}$ as follows:

$$
\begin{gathered}
{\left[\frac{d}{d r}+\frac{\kappa}{r}-U(r)\right] F_{n k}(r)=\left(M+E_{n k}-\Delta(r)\right) G_{n k}(r)} \\
{\left[\frac{d}{d r}-\frac{\kappa}{r}+U(r)\right] G_{n k}(r)=\left(M-E_{n k}+\sum(r)\right) F_{n k}(r)}
\end{gathered}
$$

where

$$
\Delta(r)=V(r)-S(r), \quad \sum(r)=V(r)+S(r)
$$

By eliminating $F_{n k}(r)$ and $G_{n k}(r)$ from Eqs. (8) and (9), the following Schrodinger-like differential equations for the upper and lower radial spinor components are obtained as follow:

$$
\begin{aligned}
& {\left[\frac{d^{2}}{d r}-\frac{\kappa(\kappa+1)}{r^{2}}+\frac{2 \kappa U(r)}{r}-\frac{d U(r)}{d r}-U^{2}(r)\right] F_{n k}(r)+\frac{\frac{d \Delta(r)}{d r}}{M+E_{n k}-\Delta(r)}\left[\frac{d}{d r}+\frac{\kappa}{r}-U(r)\right] F_{n k}(r)} \\
& =\left[\left(M+E_{n k}-\Delta(r)\right)\left(M-E_{n k}-\sum(r)\right)\right] F_{n k}(r) \\
& {\left[\frac{d^{2}}{d r}-\frac{\kappa(\kappa-1)}{r^{2}}+\frac{2 \kappa U(r)}{r}+\frac{d U(r)}{d r}-U^{2}(r)\right] G_{n k}(r)+\frac{\frac{d \sum(r)}{d r}}{M+E_{n k}-\sum(r)}\left[\frac{d}{d r}-\frac{\kappa}{r}+U(r)\right] G_{n k}(r)} \\
& =\left[\left(M+E_{n k}-\Delta(r)\right)\left(M-E_{n k}-\sum(r)\right)\right] G_{n k}(r)
\end{aligned}
$$

where $\kappa(\kappa+1)=\tilde{l}(\tilde{l}+1)$ and $\kappa(\kappa-1)=l(l+1)$. The relationship between the quantum number $\kappa$ and the quantum numbers for spin $l$ and pseudospin $\tilde{l}$ symmetries are

$$
\kappa=\left\{\begin{array}{l}
-(l+1)=-\left(j+\frac{1}{2}\right)\left(s_{\frac{1}{2}}, p_{\frac{3}{2}}\right) \\
j=l+\frac{1}{2} ; \text { aligned spin }(\kappa<0) \\
(\tilde{l}+1)=\left(j+\frac{1}{2}\right)\left(d_{\frac{3}{2}}, d_{\frac{5}{2}}\right) \\
l=l-\frac{1}{2} ; \text { unaligned } \operatorname{spin}(\kappa>0)
\end{array}\right.
$$




$$
\kappa=\left\{\begin{array}{l}
-\tilde{l}=-\left(j+\frac{1}{2}\right)\left(s_{\frac{1}{2}}, p_{\frac{3}{2}}\right) \\
j=l-\frac{1}{2} ; \text { aligned spin }(\kappa<0) \\
(\tilde{l}+1)=\left(j+\frac{1}{2}\right)\left(d_{\frac{3}{2}}, d_{\frac{5}{2}}\right) \\
j=\tilde{l}-\frac{1}{2} ; \text { unaligned spin }(\kappa>0)
\end{array} .\right.
$$

However, for spin symmetry limit, $\Delta(r)$ is a constant that is $c_{s}=\frac{d \Delta(r)}{d r}=0$ whereas for pseudospin symmetry limit $\sum(r)$ is a constant such that $c_{p s}=\frac{d \sum(r)}{d r}=0$

\section{The parametric Nikiforov-Uvarov (NU) method}

The NU method is based on reducing second order linear differential equation to a generalised equation of hypergeometric type and provides exact solutions in terms of special orthogonal functions like Jacobi and Laguerre as well as corresponding energy eigenvalues ${ }^{73-78}$. The standard differential equation for parametric NU method according to Tezcan and Sever ${ }^{79}$ is given as

$$
\psi^{\prime \prime}(s)+\frac{\left(c_{1}-c_{2} s\right)}{s\left(1-c_{3} s\right)} \psi^{\prime}(s)+\frac{1}{s^{2}\left(1-c_{3} s\right)^{2}}\left[-\Omega_{1} s^{2}+\Omega_{2} s-\Omega_{3}\right] \psi(s)=0
$$

The parametric constants are obtained as follows

$$
\left.\begin{array}{l}
c_{4}=\frac{1}{2}\left(1-c_{1}\right) ; \quad c_{5}=\frac{1}{2}\left(c_{2}-c_{3}\right) ; \quad c_{6}=c_{5}^{2}+\epsilon_{1} \\
c_{7}=2 c_{4} c_{5}-\Omega_{2} ; c_{8}=c_{4}^{2}+\Omega_{3} ; \quad c_{9}=c_{3} c_{7}+c_{3}^{2} c_{8}+c_{6} \\
c_{10}=c_{1}+2 c_{4}+2 \sqrt{c_{8}} ; \quad c_{11}=c_{2}-2 c_{5}+2\left(\sqrt{c_{9}}+c_{3} \sqrt{c_{8}}\right) \\
c_{12}=c_{4}+\sqrt{c_{8}} ; \quad c_{13}=c_{5}-\left(\sqrt{c_{9}}+c_{3} \sqrt{c_{8}}\right)
\end{array}\right\} .
$$

The condition for energy equation is given as

$$
c_{2} n-(2 n+1) c_{5}(2 n+1)\left(\sqrt{c_{9}}+c_{3} \sqrt{c_{8}}\right)+n(n-1) c_{3}+c_{7}+2 c_{3} c_{8}+2 \sqrt{c_{8} c_{9}}=0
$$

The corresponding total wave function is then given as

$$
\Psi(s)=N_{n l} s^{c_{12}}\left(1-c_{3} s\right)^{-c_{12}-\frac{c_{11}}{c_{3}}} P_{n}^{\left(c_{10}-1, \frac{c_{11}}{c_{3}}-c_{10}-1\right)}\left(1-2 c_{3} s\right) .
$$

\section{Solution of Dirac equation with the proposed potential}

Dirac equation ${ }^{8}$ with equal scalar and vector potential without tensor interaction is given as

$$
\left[\frac{d^{2}}{d r^{2}}-\frac{k(k+1)}{r^{2}}-2\left(E_{n l}+M\right) V(r)+\left(E_{n l}^{2}-M^{2}\right)\right] F_{n k}=0
$$

Equation (19) can further be express as

$$
\frac{d^{2} F_{n k}}{d r^{2}}+\left[-\frac{k(k+1)}{r^{2}}-2 \gamma V(r)+\beta^{2}\right] F_{n k}=0
$$

where

$$
\gamma=\left(E_{n l}^{2}-M^{2}\right), \beta=\left(E_{n l}^{2}-M^{2}\right) .
$$

The hyperbolic Hulthen plus hyperbolic exponential inversely quadratic potential is given as

$$
V(r)=\left[\frac{v_{1} e^{-\frac{r}{b}} \cosh \omega}{1-e^{-\frac{r}{b}} \cosh \omega}-\frac{A e^{-\frac{r}{b}} \cosh \omega}{r^{2}}\right]
$$

where $v_{1}$ is the potential depth, $A$ is a real constant parameter, $b$ is the screening parameter representing the strength of the potential and $\omega$ is the optimising parameter. The potential of Eq. (22) can be express as

$$
V(r)=\left[\frac{v_{1} q e^{-v r}}{1-q e^{-v r}}-\frac{A q e^{-v r}}{r^{2}}\right]
$$

where $v=\frac{1}{b}, q=\cosh \omega$. The standard Greene-Aldrich approximation to the centrifugal term suitable for the propose potential is given as

$$
\frac{1}{r^{2}}=\frac{v^{2} q e^{-v r}}{\left(1-q e^{-v r}\right)}
$$

Substituting (23) and (24) into (20) and simplifying gives 


$$
\frac{d^{2} F_{n k}}{d r^{2}}+\left[-\frac{k(k+1) v^{2} q e^{-v r}}{\left(1-q e^{-v r}\right)^{2}}+\frac{2 \gamma v_{1} q e^{-v r}}{\left(1-q e^{-v r}\right)}+\frac{2 A \gamma v^{2} q^{2} e^{-2 v r}}{\left(1-q e^{-v r}\right)^{2}}+\beta^{2}\right] F_{n k}=0
$$

Let $s=e^{-v r}$, then (25) reduces to

$$
\begin{aligned}
& \frac{d^{2} F_{n k}}{d r^{2}}+\frac{(1-q s)}{s(1-q s)} \frac{d F_{n k}}{d s}+\frac{1}{s^{2}(1-s)^{2}}\left[-\left(\frac{2 \gamma v_{1} q^{2}}{v^{2}}-2 A \gamma q^{2}-\frac{q^{2} \beta^{2}}{v^{2}}\right) s^{2}\right. \\
& \left.+\left(\kappa(\kappa+1) q-\frac{2 q \beta^{2}}{v^{2}}+\frac{2 \gamma v_{1} q}{v^{2}}\right) s-\left(-\frac{\beta^{2}}{v^{2}}\right)\right] F_{n k}=0
\end{aligned}
$$

Comparing (26)-(15), the followings are obtain

$$
\left[\Omega_{1}=\left(\frac{2 \gamma v_{1} q^{2}}{v^{2}}-2 A \gamma q^{2}-\frac{q^{2} \beta^{2}}{v^{2}}\right), \Omega_{2}=\left(\kappa(\kappa+1) q-\frac{2 q \beta^{2}}{v^{2}}+\frac{2 \gamma v_{1} q}{v^{2}}\right), \Omega_{3}=\left(-\frac{\beta^{2}}{v^{2}}\right)\right]
$$

Using Eq. (16), the following parametric constants are obtained as follows:

$$
\left.\begin{array}{l}
c_{1}=1, \quad c_{2}=c_{3}=q ; \quad c_{4}=0 ; \quad c_{5}=-\frac{q}{2} ; \quad c_{6}=\frac{q^{2}}{4}+\frac{2 \gamma v_{1} q^{2}}{v^{2}}-2 A \gamma q^{2}-\frac{q^{2} \beta^{2}}{v^{2}} \\
c_{7}=-\kappa(\kappa+1) q+\frac{2 q \beta^{2}}{v^{2}}-\frac{2 \gamma v^{2} q}{v^{2}} ; \quad c_{8}=-\frac{\beta^{2}}{v^{2}} ; \quad c_{9}=\kappa(\kappa+1) q^{2}+\frac{q^{2}}{4}-2 \gamma q^{2} A \\
c_{10}=1+2 \sqrt{-\frac{\beta^{2}}{v^{2}} ;} \quad c_{11}=2 q+2 \sqrt{4 \kappa(\kappa+1) q^{2}-8 A \gamma q^{2}+q^{2}}+2 q \sqrt{-\beta^{2} b^{2}} \\
c_{12}=\sqrt{-\beta^{2} b^{2}} ; \quad c_{13}=-\frac{q}{2}-\left[\frac{1}{2} \sqrt{4 \kappa(\kappa+1) q^{2}-8 A \gamma q^{2}+q^{2}}+2 q \sqrt{-\beta^{2} b^{2}}\right]
\end{array}\right\} .
$$

Using Eq. (17) with some algebraic simplification, we have

$$
\beta^{2}=-\frac{1}{b^{2}}\left[\frac{\left(n^{2}+n+\frac{1}{2}\right) q+q\left(n+\frac{1}{2}\right) \sqrt{(2 \kappa+1)^{2}-8 A \gamma}+q \kappa(\kappa+1)-2 q \gamma v_{1} b^{2}}{(2 n+1) q+q \sqrt{(2 \kappa+1)^{2}-8 A \gamma}}\right]
$$

substituting (21)-(29) gives the relativistic spin energy equation as

$$
\left(E_{n k}^{s}-M\right)\left(E_{n k}^{s}+M\right)=-\frac{1}{b^{2}}\left[\begin{array}{c}
\left(n^{2}+n+\frac{1}{2}\right) q+q\left(n+\frac{1}{2}\right) \sqrt{(2 \kappa+1)^{2}-8 A\left(E_{n k}^{s}+M\right)} \\
+q \kappa(\kappa+1)-2 q\left(E_{n k}^{s}+M\right) v_{1} b^{2} \\
(2 n+1) q+q \sqrt{(2 \kappa+1)^{2}-8 A\left(E_{n k}^{s}+M\right)}
\end{array}\right]^{2} .
$$

Equation (30) can also be express as

$$
\left(E_{n k}^{s}-M\right)\left(E_{n k}^{s}+M\right)=-\frac{1}{b^{2}}\left[\begin{array}{c}
\left(n^{2}+n+\frac{1}{2}\right) \cosh \omega \\
+\left(n+\frac{1}{2}\right) \sqrt{(2 \kappa+1)^{2}-8 A\left(E_{n k}^{s}+M\right)} \cosh \omega \\
+\kappa(\kappa+1) \cosh \omega-2\left(E_{n k}^{s}+M\right) v_{1} b^{2} \cosh \omega
\end{array}\right]
$$

In order to obtain the solution for the pseudospin symmetry, then the condition for pseudospin symmetry limit discussed in "Dirac equation for spin and pseudospin symmetry" section is fully applied such that $\gamma=E_{n k}-M-c_{p s}, \beta=\left(M+E_{n k}-c_{p s}\right), c_{p s}=0$. Following the same steps to obtain the energy equation for the spin symmetry, the energy equation for pseudospin symmetry limit is then given as

$$
\left(M+E_{n k}^{p s}\right)\left(M-E_{n k}^{p s}\right)=-\frac{1}{b^{2}}\left[\begin{array}{c}
\left(n^{2}+n+\frac{1}{2}\right) q+q\left(n+\frac{1}{2}\right) \sqrt{(1-2 \kappa)^{2}-8 A\left(E_{n k}^{s}+M\right)} \\
+q \kappa(\kappa-1)-2 q\left(E_{n k}^{p s}-M\right) v_{1} b^{2} \\
(2 n+1) q+q \sqrt{(1-2 \kappa)^{2}-8 A\left(E_{n k}^{p s}-M\right)}
\end{array}\right]^{2} .
$$

Using Eq. (8), the upper component of the wave function is given as

$$
\begin{aligned}
& \Psi_{n l}=N_{n l}\left(e^{-\frac{r}{b}} \cosh \omega\right)^{\sqrt{-\beta^{2} b^{2}}}\left(1-e^{-\frac{r}{b}} \cosh \omega\right)^{\eta} \\
& \times P_{n}^{\left[\sqrt{-\beta^{2} b^{2}}, 2 \cosh \omega+2 \sqrt{4 \kappa(\kappa+1)(\cosh \omega)^{2}-8 A \gamma(\cosh \omega)^{2}+(\cosh \omega)^{2}}+2 \cosh \omega \sqrt{-\beta^{2} b^{2}}-2 \sqrt{-\beta^{2} b^{2}}-2\right]} \\
& \times\left(1-2 e^{-\frac{r}{b}} \cosh \omega\right)
\end{aligned}
$$

where 


$$
\begin{aligned}
\eta= & -\sqrt{-\beta^{2} b^{2}}-\left[2 \cosh \omega+2 \sqrt{4 \kappa(\kappa+1)(\cosh \omega)^{2}-8 A \gamma(\cosh \omega)^{2}+(\cosh \omega)^{2}}\right. \\
& \left.+2 \cosh \omega \sqrt{-\beta^{2} b^{2}}\right] .
\end{aligned}
$$

The lower component of the Dirac spinor can be evaluated using the equation

$$
G_{n k}(r)=\frac{1}{M+E_{n k}}\left[\frac{d}{d r}+\frac{k}{r}\right] F_{n k}(r)
$$

where $E_{n k} \neq-M+c_{s}$

Non-relativistic solution. The non-relativistic(NR) solution of the spin symmetry leads to the Schrodinger-like solution. This is obtain by applying non-relativistic limit to the relativistic spin energy equation (30). The non relativistic transformation equations are $M+E_{n k}=\frac{2 \mu}{\hbar^{2}}, M-E_{n k}=-E_{n l}$ as $\kappa \rightarrow l$. Substituting above condition into (31) and simplifying gives the nonrelativistic energy eigen equation as

$$
E_{n l}=-\frac{\hbar^{2}}{2 \mu b^{2}}\left[\frac{q\left(n^{2}+n+\frac{1}{2}\right)+q\left(n+\frac{1}{2}\right) \sqrt{(2 l+1)^{2}-\frac{8 \mu A}{\hbar^{2}}}+q l(l+1)-\frac{2 q \mu v_{1} b^{2}}{\hbar^{2}}}{(2 n+1) q+q \sqrt{(2 l+1)^{2}-\frac{8 \mu A}{\hbar^{2}}}}\right]^{2} .
$$

\section{Deduction for special cases.}

(a) Hulthen potential: Substituting $A=0, q=1$ into (23), the potential reduces to Hulthen potential.

$$
V(r)=-\frac{v_{1} e^{-\frac{r}{b}}}{\left(1-e^{-\frac{r}{b}}\right)}
$$

The resulting energy equation is given as

$$
E_{n l}=-\frac{\hbar^{2}}{2 \mu b^{2}}\left[\frac{(1+n+1)}{2}-\frac{\frac{2 \mu v_{1} b^{2}}{\hbar^{2}}}{2(1+n+l)}\right]^{2}
$$

However, for the purpose of comparison of the Hulthen potential to an existing literature, let $v_{1}=\frac{z e^{2}}{b}$ where $z=e=1$ in atomic mass unit. Substituting $v_{1}$ into Eq. (37) gives the energy equation of the Hulthen potential as

$$
E_{n l}=-\frac{\hbar^{2}}{2 \mu b^{2}}\left[\frac{(1+n+1)}{2}-\frac{\frac{2 \mu b}{\hbar^{2}}}{2(1+n+l)}\right]^{2}
$$

(b) Exponential inversely quadratic potential: When $v_{1}=0$, and $q=1$, the potential reduces to exponential inversely quadratic potential

$$
V(r)=\frac{-A e^{-\frac{r}{b}}}{r^{2}}
$$

The resulting energy equation is given as

$$
E_{n l}=-\frac{\hbar^{2}}{2 \mu b^{2}}\left[\frac{\left(n^{2}+n+\frac{1}{2}\right)+\left(n+\frac{1}{2}\right) \sqrt{(2 l+1)^{2}-\frac{8 \mu A}{\hbar^{2}}}+l(l+1)}{(2 n+1)+\sqrt{(2 l+1)^{2}-\frac{8 \mu A}{\hbar^{2}}}}\right]^{2} .
$$

\section{Thermodynamic properties for the potential model}

The thermodynamic properties for the potential model is calculated using Eq. (35) for $q=1$. The thermodynamic properties for a quantum mechanical systems can be obtained from the exact partition function given by

$$
Z(\beta)=\sum_{n=0}^{\lambda} e^{-\beta E_{n}}
$$

where $\lambda$ is an upper bound of the vibrational quantum number obtained from the numerical solution of $\frac{d E_{n}}{d n}=0$, $\beta=\frac{1}{k T}$ where $K$ and $T$ are Boltzmann constant and absolute temperature respectively. In the classical limit, the summation in (41) can be replaced with the integral: 


$$
Z(\beta)=\int_{0}^{\lambda} e^{-\beta E_{n}} d n
$$

The energy equation of Eq. (35) can be simplified to

$$
E_{n l}=-Q_{1}\left[(n+\Delta)+\frac{Q_{2}}{(n+\Delta)}\right]^{2}
$$

where

$$
Q_{1}=\frac{\hbar^{2}}{8 \mu b^{2}}, Q_{2}=\frac{2 \mu}{\hbar^{2}}\left(A-V_{1} b^{2}\right), \Delta=\frac{1}{2}+\frac{1}{2} \sqrt{\left(l+\frac{1}{2}\right)^{2}-\frac{2 \mu A}{\hbar^{2}}}
$$

The maximum vibrational quantum number is given by $\lambda=-\Delta+\sqrt{Q_{2}}$. The energy equation (43) can then be express in the form

$$
E_{n l}=-\left[Q_{1} \rho^{2}+\frac{Q_{1} Q_{2}^{2}}{\rho^{2}}\right]-2 Q_{1} Q_{2}, \rho=n+\Delta
$$

Hence, the partition function Eq. (42) can be express in the classical limit as

$$
Z(\beta)=e^{2 \beta Q_{1} Q_{2}} \int_{0}^{\lambda} e^{\left(Q_{1} \rho^{2}+\frac{Q_{1} Q_{2}^{2}}{\rho^{2}}\right)} d \rho
$$

Equation (46) is integrated using MAPLE package. Hence, the integral equation (46) which is the partition function is given as

$$
Z(\beta)=e^{2 \beta Q_{1} Q_{2}} \sqrt{\pi}\left[\begin{array}{c}
e^{2 \beta Q_{1} Q_{2}} \operatorname{erf}\left(\sqrt{-\beta Q_{1}} \lambda+\frac{Q_{2} \sqrt{-\beta Q_{1}}}{\lambda}\right) \\
+e^{-2 \beta Q_{1} Q_{2}} \operatorname{erf}\left(\sqrt{-\beta Q_{1}} \lambda-\frac{Q_{2} \sqrt{-\beta Q_{1}}}{\lambda}\right) \\
4 \sqrt{-\beta Q_{1}}
\end{array}\right]
$$

Using the partition function (47), other thermodynamic properties are obtain as follows

(a) Vibrational mean energy:

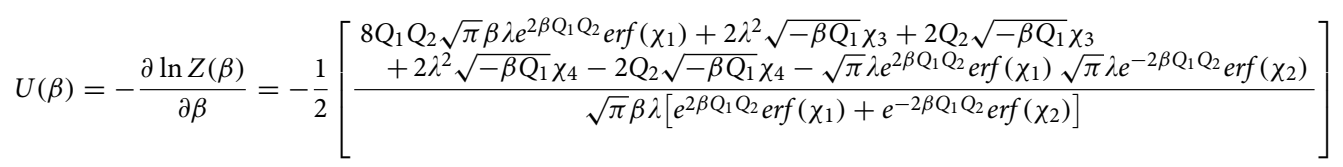

where

$$
\left.\begin{array}{l}
\chi_{1}=\left(\frac{\sqrt{-\beta Q_{1}}\left(\lambda^{2}+Q_{2}\right)}{\lambda}\right) \\
\chi_{2}=\left(\frac{\sqrt{-\beta Q_{1}}\left(\lambda^{2}-Q_{2}\right)}{\lambda}\right) \\
\chi_{3}=e^{\frac{\beta Q_{1}\left(4 Q_{2} \lambda^{2}+\lambda^{4}+Q_{2}^{2}\right)}{\lambda^{2}}} \\
\chi_{4}=e^{\frac{\beta Q_{1}\left(-4 Q_{2} \lambda^{2}+\lambda^{4}+Q_{2}^{2}\right)}{\lambda^{2}}}
\end{array}\right\}
$$

(b) Vibrational specific heat capacity:

$$
C(\beta)=k \beta^{2}\left(\frac{\partial^{2} \ln Z(\beta)}{\partial \beta^{2}}\right)=\frac{1}{2} k\left[\frac{\chi_{9}+\chi_{10}+\chi_{11}+\chi_{12}+\chi_{13}}{\sqrt{-\beta Q_{1}} \pi \lambda^{3}\left[e^{2 \beta Q_{1} Q_{2}} \operatorname{erf}\left(\chi_{1}\right)+e^{-2 \beta Q_{1} Q_{2}} \operatorname{erf}\left(\chi_{2}\right)\right]^{2}}\right]
$$

where 


$$
\begin{aligned}
& \chi_{5}=e^{\frac{\beta Q_{1}\left(6 Q_{2} \lambda^{2}+\lambda^{4}+Q_{2}^{2}\right)}{\lambda^{2}}} \\
& \chi_{6}=e^{\frac{\beta Q_{1}\left(-6 Q_{2} \lambda^{2}+\lambda^{4}+Q_{2}^{2}\right)}{\lambda^{2}}} \\
& \chi_{7}=e^{\frac{2 \beta Q_{1}\left(4 Q_{2} \lambda^{2}+\lambda^{4}+Q_{2}^{2}\right)}{\lambda^{2}}} \\
& \chi_{8}=e^{\frac{2 \beta Q_{1}\left(-4 Q_{2} \lambda^{2}+\lambda^{4}+Q_{2}^{2}\right)}{\lambda^{2}}} \\
& \chi_{9}=-\beta Q_{1} Q_{2} \lambda^{2} \sqrt{\pi} e^{\frac{\beta Q_{1}\left(\lambda^{2}+Q_{2}\right)^{2}}{\lambda^{2}}} \operatorname{erf}\left(\chi_{2}\right)+\beta Q_{1} Q_{2} \lambda^{2} \sqrt{\pi} e^{\frac{\beta Q_{1}\left(\lambda^{2}-Q_{2}\right)^{2}}{\lambda^{2}}} \operatorname{erf}\left(\chi_{1}\right) \\
& -2 Q_{1} \lambda^{5} \beta \sqrt{-\beta Q_{1}} \chi_{8} \\
& -\lambda^{3} \pi \sqrt{-\beta Q_{1}} e^{4 \beta Q_{1} Q_{2}} \operatorname{erf}\left(\chi_{1}\right)^{2}-\lambda^{3} \pi \sqrt{-\beta Q_{1}} e^{-4 \beta Q_{1} Q_{2}} \operatorname{erf}\left(\chi_{1}\right)^{2} \\
& -4 Q_{1} Q_{2} \lambda^{3} \beta \sqrt{-\beta Q_{1}} \chi_{3}+4 Q_{1} Q_{2} \lambda^{3} \beta \sqrt{-\beta Q_{1}} \chi_{8}-2 Q_{1} Q_{2}^{2} \lambda \beta \sqrt{-\beta Q_{1}} \chi_{7} ; \\
& \chi_{10}=4 Q_{1} Q_{2}^{2} \lambda \beta \sqrt{-\beta Q_{1}} e^{\frac{2 \beta Q_{1}\left(\lambda^{4}+Q_{2}^{2}\right)}{\lambda^{2}}}-2 Q_{1} Q_{2}^{2} \lambda \beta \sqrt{-\beta Q_{1}} \chi_{8} \\
& -\beta Q_{1} \lambda^{4} \sqrt{\pi} e^{\frac{\beta Q_{1}\left(\lambda^{2}+Q_{2}\right)^{2}}{\lambda^{2}}} \operatorname{erf}\left(\chi_{2}\right)-\beta Q_{1} \lambda^{4} \sqrt{\pi} e^{\frac{\beta Q_{1}\left(\lambda^{2}-Q_{2}\right)^{2}}{\lambda^{2}}} \operatorname{erf}\left(\chi_{1}\right) \\
& +2 Q_{1}^{2} \sqrt{\pi} \beta^{2} \lambda^{6} e^{\frac{\beta Q_{1}\left(\lambda^{2}+Q_{2}^{2}\right)}{\lambda^{2}}} \text {. } \\
& \chi_{11}=2 Q_{1}^{2} Q_{2}^{3} \sqrt{\pi} \beta^{2} e^{\frac{\beta Q_{1}\left(\lambda^{2}+Q_{2}\right)^{2}}{\lambda^{2}}} \operatorname{erf}\left(\chi_{2}\right)+2 Q_{1}^{2} \lambda^{6} e^{\frac{\beta Q_{1}\left(\lambda^{2}+Q_{2}\right)^{2}}{\lambda^{2}}} \operatorname{erf}\left(\chi_{1}\right) \\
& -2 Q_{1}^{2} Q_{2}^{3} \beta^{2} e^{\frac{\beta Q_{1}\left(\lambda^{2}+Q_{2}\right)^{2}}{\lambda^{2}}} \operatorname{erf}\left(\chi_{1}\right)+2 Q_{1}^{2} \sqrt{\pi} \beta^{2} \chi_{5} \lambda^{6} \operatorname{erf}\left(\chi_{1}\right)+2 Q_{1}^{2} \sqrt{\pi} \beta^{2} \chi_{5} \lambda^{6} \operatorname{erf}\left(\chi_{2}\right) \\
& -2 Q_{1}^{2} Q_{2}^{3} \sqrt{\pi} \beta^{2} \chi_{6} \operatorname{erf}\left(\chi_{2}\right) \\
& \chi_{12}=-2 \sqrt{-\beta Q_{1}} \lambda^{3} \pi \operatorname{erf}\left(\chi_{1}\right) \operatorname{erf}\left(\chi_{2}\right)-2 Q_{1} \lambda^{5} \beta \sqrt{-\beta Q_{1}} \operatorname{erf}\left(\chi_{3}\right) \\
& \begin{array}{l}
-4 Q_{1} \lambda^{5} \beta \sqrt{-\beta Q_{1}} e^{\frac{2 \beta Q_{1}\left(\lambda^{4}+Q_{2}^{2}\right)}{\lambda^{2}}}-\beta Q_{1} Q_{2} \lambda^{2} \sqrt{\pi} \chi_{6} \operatorname{erf}\left(\chi_{2}\right) \\
+6 Q_{1}^{2} \sqrt{\pi} \beta^{2} \lambda^{4} \chi_{5} Q_{2} \operatorname{erf}\left(\chi_{1}\right)+6 Q_{1}^{2} \sqrt{\pi} \beta^{2} \lambda^{2} \chi_{5} Q_{2}^{2} \operatorname{erf}\left(\chi_{1}\right)
\end{array} \\
& \chi_{13}=-6 Q_{1}^{2} \sqrt{\pi} \beta^{2} \chi_{6} \lambda^{4} Q_{2} \operatorname{erf}\left(\chi_{2}\right)-32 Q_{1}^{2} \pi \beta^{2} \lambda^{3} Q_{2}^{2} \operatorname{erf}\left(\chi_{1}\right) \sqrt{-\beta Q_{1}} \operatorname{erf}\left(\chi_{2}\right) \\
& +6 Q_{1}^{2} \sqrt{\pi} \beta^{2} \lambda^{2} \chi_{6} Q_{2}^{2} \operatorname{erf}\left(\chi_{2}\right)+22 \lambda^{2} Q_{1}^{2} Q_{2}^{2} \beta^{2} \sqrt{\pi} e^{\frac{\beta Q_{1}\left(\lambda^{2}+Q_{2}\right)^{2}}{\lambda^{2}}} \operatorname{erf}\left(\chi_{2}\right) \\
& -22 \lambda^{4} Q_{1}^{2} Q_{2} \beta^{2} \sqrt{\pi} e^{\frac{\beta Q_{2}\left(\lambda^{2}+Q_{2}\right)^{2}}{\lambda^{2}}} \operatorname{erf}\left(\chi_{1}\right)
\end{aligned}
$$

(c) Vibrational entropy

$$
\begin{aligned}
S(\beta)= & k \ln Z(\beta)-k \beta \frac{\partial \ln Z(\beta)}{\partial \beta}=k \ln e^{2 \beta Q_{1} Q_{2}} \sqrt{\pi}\left[\begin{array}{c}
e^{2 \beta Q_{1} Q_{2}} \operatorname{erf}\left(\sqrt{-\beta Q_{1}} \lambda+\frac{Q_{2} \sqrt{-\beta Q_{1}}}{\lambda}\right) \\
+e^{-2 \beta Q_{1} Q_{2}} \operatorname{erf}\left(\sqrt{-\beta Q_{1}} \lambda-\frac{Q_{2} \sqrt{-\beta Q_{1}}}{\lambda}\right) \\
4 \sqrt{-\beta Q_{1}}
\end{array}\right] \\
& -\frac{1}{2} k \beta\left[\begin{array}{c}
8 Q_{1} Q_{2} \sqrt{\pi} \beta \lambda e^{2 \beta Q_{1} Q_{2}} \operatorname{erf}\left(\chi_{1}\right)+2 \lambda^{2} \sqrt{-\beta Q_{1}} \chi_{3}+2 Q_{2} \sqrt{-\beta Q_{1}} \chi_{3} \\
+2 \lambda^{2} \sqrt{-\beta Q_{1}} \chi_{4}-2 Q_{2} \sqrt{-\beta Q_{1}} \chi_{4}-\sqrt{\pi} \lambda e^{2 \beta Q_{1} Q_{2}} \operatorname{erf}\left(\chi_{1}\right) \sqrt{\pi} \lambda e^{-2 \beta Q_{1} Q_{2}} \operatorname{erf}\left(\chi_{2}\right) \\
\sqrt{\pi} \beta \lambda\left[e^{2 \beta Q_{1} Q_{2}} \operatorname{erf}\left(\chi_{1}\right)+e^{-2 \beta Q_{1} Q_{2}} \operatorname{erf}\left(\chi_{2}\right)\right]
\end{array}\right]
\end{aligned}
$$

(d) Vibrational free energy

$$
F(\beta)=-k T \ln Z(\beta)=-\frac{1}{\beta} \ln e^{2 \beta Q_{1} Q_{2}} \sqrt{\pi}\left[\begin{array}{c}
e^{2 \beta Q_{1} Q_{2}} \operatorname{erf}\left(\sqrt{-\beta Q_{1}} \lambda+\frac{Q_{2} \sqrt{-\beta Q_{1}}}{\lambda}\right) \\
+e^{-2 \beta Q_{1} Q_{2}} \operatorname{erf}\left(\sqrt{-\beta Q_{1}} \lambda-\frac{Q_{2} \sqrt{-\beta Q_{1}}}{\lambda}\right) \\
4 \sqrt{-\beta Q_{1}}
\end{array}\right] .
$$

\section{Numerical results}

Using Maple software package, the numerical solutions for spin and pseudospin symmetries were obtained using Eqs. (30) and (32) respectively. the nonrelativistic numerical bound state solutions were obtained using (35). Finally, the numerical solutions for Hulthen potential were obtained using (38) as one of the special cases in comparison to the results of existing literature.

\section{Discussion}

Figure 1 is the graph of standard Greene-Aldrich approximation to the centrifugal term. The trend of Fig. 1 shows that the approximation was suitable and appropriate for the proposed potential. Figure $2 \mathrm{a}, \mathrm{b}$ are the partition function $Z(\lambda, \beta)$ which increases exponentially with respect to $\lambda$ and $\beta$ respectively. The vibrational mean energy $U(\lambda, \beta)$ curves are shown in Fig. $2 \mathrm{c}$,d. The vibrational mean energy decreases with temperature parameter 


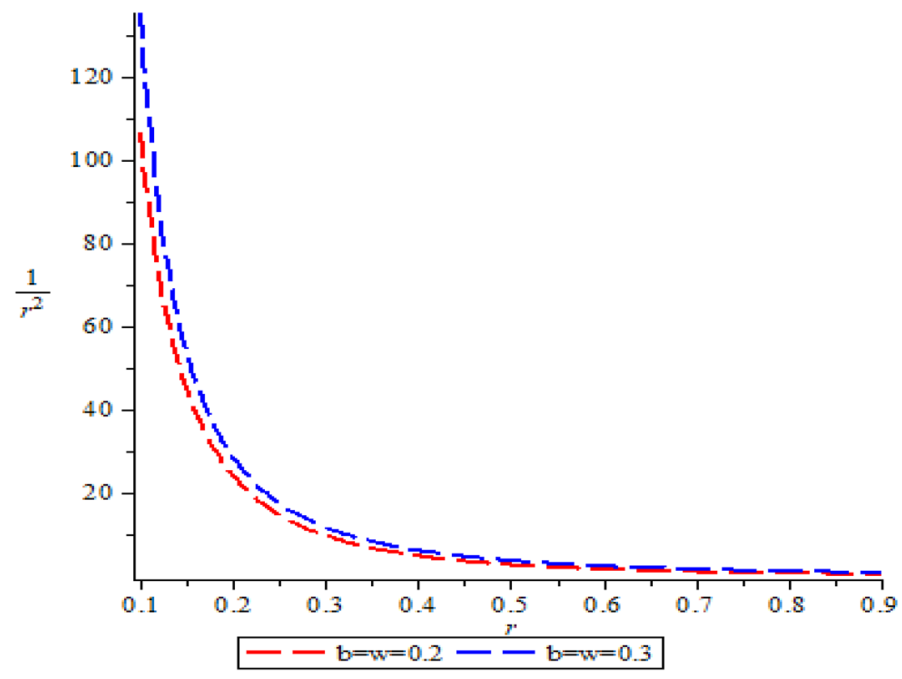

Figure 1. Standard Greene-Aldrich approximation to the centrifugal term.

$\beta$, but increases monotonically with a maximum turning point at $\lambda=4$ with respect to $\lambda$. The vibrational specific heat capacities $C(\lambda, \beta)$ is shown in Fig. $2 \mathrm{e}, \mathrm{f}$, with $\lambda$; it has a perfect curve with a minimum turning point at different values of $\beta$. However, with $\beta$, it deceases and rises to a certain value and then decreases monotonically. The vibrational entropy $S(\lambda, \beta)$ decreases with an increasing $\lambda$ and $\beta$ as shown in Fig. $2 \mathrm{~g}$, h respectively. The vibrational free energy $F(\lambda, \beta)$ presented in Fig. 2i,j increases with increasing $\lambda$ and $\beta$ respectively. The behaviour of the thermal properties in the present study are in accordance with those reported in refs ${ }^{68,71,72}$. Table 1 is the numerical values for spin symmetry energies for $A=5 \mathrm{fm}^{-1}, V_{1}=15 \mathrm{fm}^{-1}, b=0.1$ and $M=10 \mathrm{fm}^{-1}$. Table 2 is the numerical values for spin symmetry energies for $A=5 \mathrm{fm}^{-1}, V_{1}=15 \mathrm{fm}^{-1}, b=0.2$ and $M=10 \mathrm{fm}^{-1}$. Table 3 is the numerical values for spin symmetry energies for $A=5 \mathrm{fm}^{-1}, V_{1}=15 \mathrm{fm}^{-1}, b=0.3$ and $M=10 \mathrm{fm}^{-1}$ while Table 4 is the numerical values for spin symmetry energies for $A=5 \mathrm{fm}^{-1}, V_{1}=15 \mathrm{fm}^{-1}, b=0.3$ and $M=10 \mathrm{fm}^{-1}$. In Table 2, the S-spin quantum state $0 s_{\frac{1}{2}}$ has the highest relativistic spin energy symmetry value of $10.65566240 \mathrm{fm}^{-1}$ for $\kappa<0$ and the minimum value of $-9.402539935 \mathrm{fm}^{-1}$ for $\kappa>0$. In Table 3, the S-spin quantum state $0 s_{\frac{1}{2}}$ has the highest relativistic spin energy symmetry value of $11.94562892 \mathrm{fm}^{-1}$ for $\kappa<0$ and the minimum value of $-9.653034948 \mathrm{fm}^{-1}$ for $\kappa>0$. In Table 4 , the S-spin quantum state $0 s_{\frac{1}{2}}$ has the highest relativistic spin energy symmetry value of $13.56961400 \mathrm{fm}^{-1}$ for $\kappa<0$ and the minimum value of $-9.759490473 \mathrm{fm}^{-1}$ for $\kappa>0$. This illustrates that the s-spin quantum state assume the most stable state for relativistic spin energy symmetry. Also, Tables 1, 2, 3 and 4 are predominantly positive values which are applicable in describing some properties of relativistic antiparticles. Considering Tables 1, 2, 3 and4, the energy rise with an increase in the screening parameter. The following degeneracies were obtain for the screening parameter $b=0.1,0.2,0.3$ and $0.4: 3 p_{\frac{3}{2}}=3 s_{\frac{1}{2}}, 2 f_{\frac{7}{2}}=2 d_{\frac{5}{2}}, 3 f_{\frac{7}{2}}=3 d_{\frac{5}{2}}, 0 f_{\frac{7}{2}}=0 d_{\frac{5}{2}}$ and $1 f_{\frac{7}{2}}=1 d_{\frac{5}{2}}$.

Table 5 is the numerical values for pseudospin symmetry for $A=5 \mathrm{fm}^{-1}, V_{1}=15 \mathrm{fm}^{-1}, b=0.1$ and $M=10 \mathrm{fm}^{-1}$. Table 6 is the numerical values for pseudospin symmetry for $A=5 \mathrm{fm}^{-1}, V_{1}=15 \mathrm{fm}^{-1}, b=0.2$ and $M=10 \mathrm{fm}^{-1}$, Table 7 is the numerical values for pseudospin symmetry for $A=5 \mathrm{fm}^{-1}, V_{1}=15 \mathrm{fm}^{-1}, b=0.3$ and $M=10 \mathrm{fm}^{-1}$ and Table 8 is the numerical values for pseudospin symmetry for $A=5 \mathrm{fm}^{-1}, V_{1}=15 \mathrm{fm}^{-1}$, $b=0.4$ and $M=10 \mathrm{fm}^{-1}$. Tables 5, 6, 7 and 8 are the numerical relativistic pseudospin bound state energies which constitutes negative energy eigenvalues which can be use to describe the properties of relativistic particles. The degeneracies obtained for the pseudospin limit with the same screening parameter are: $3 s_{\frac{1}{2}}=3 p_{\frac{3}{2}}$, $0 p_{\frac{3}{2}}=0 d_{\frac{5}{2}}, 2 s_{\frac{1}{2}}=2 p_{\frac{3}{2}}, 3 p_{\frac{3}{2}}=3 d_{\frac{5}{2}}, 2 d_{\frac{5}{2}}=2 f_{\frac{7}{2}}, 1 d_{\frac{5}{2}}=1 f_{\frac{7}{2}}$. Figure 3 a is the variation of energy spectra for S-quantum spin symmetry. Figure $3 \mathrm{~b}$ is the variation of energy spectra for s-quantum pseudospin symmetry. Figure $3 \mathrm{c}$ is the variation of energy spectra for p-quantum spin symmetry while Fig. $3 \mathrm{~d}$ is the variation of energy spectra for P-quantum pseudospin symmetry. Considering Fig. 3a-d, there is an inverse relationship between the spin and pseudospin energy spectra. spin spectral diagram is an exact opposite of pseudospin diagram. Figure 4a is the variation of energy spectra for a mixed quantum spin state while Fig. $4 \mathrm{~b}$ is the variation of energy spectra for a mixed pseudo quantum spin. There is also an inverse relationship between the mixed spin quantum state and the mixed pseudo quantum spin as observed in Fig. 3a-d Meanwhile, the numerical bound state energies decreases with an increase in quantum state for both spin and pseudospin symmetries. Table 9 is nonrelativistic numerical bound state energies for different quantum state obtain using Eq. (35). The energy eigenvalues of Table 9 are predominantly negative which is the necessary and sufficient condition for bound state. Figure 5 a is the nonrelativistic energy spectra for the screening parameter $b=0.1$, Fig. $5 \mathrm{~b}$ is the nonrelativistic energy spectra for the screening parameter $b=0.2$, Fig. $5 \mathrm{c}$ is the nonrelativistic energy spectra for the screening parameter $b=0.3$ and Fig. $5 \mathrm{~d}$ is the nonrelativistic energy spectra for the screening parameter $b=0.4$. Figure $5 \mathrm{a}-\mathrm{d}$ show unique quantisation of different energy level which is very important concept in quantum physics in the description of atomic structure. 
a

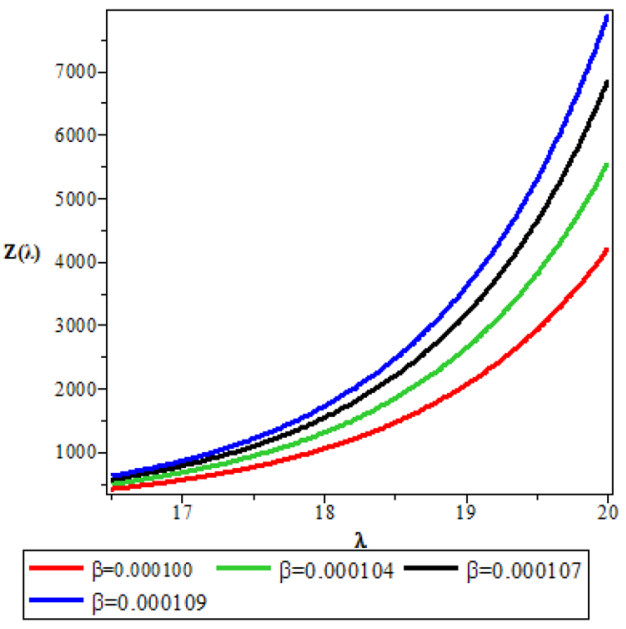

C

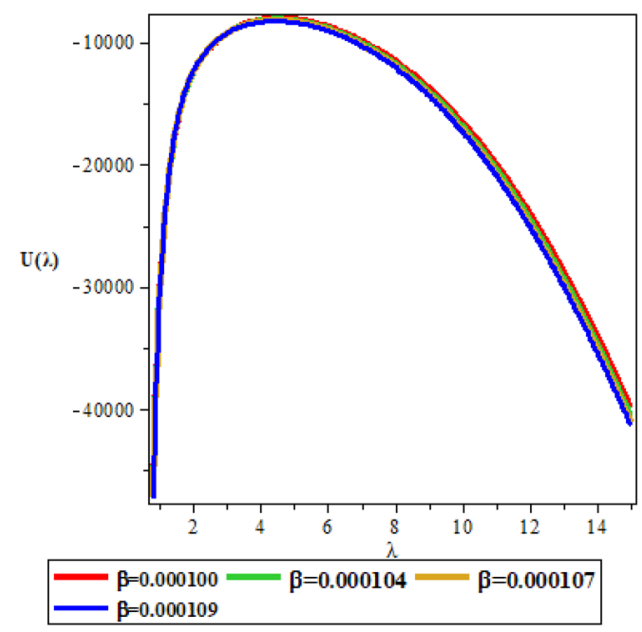

e

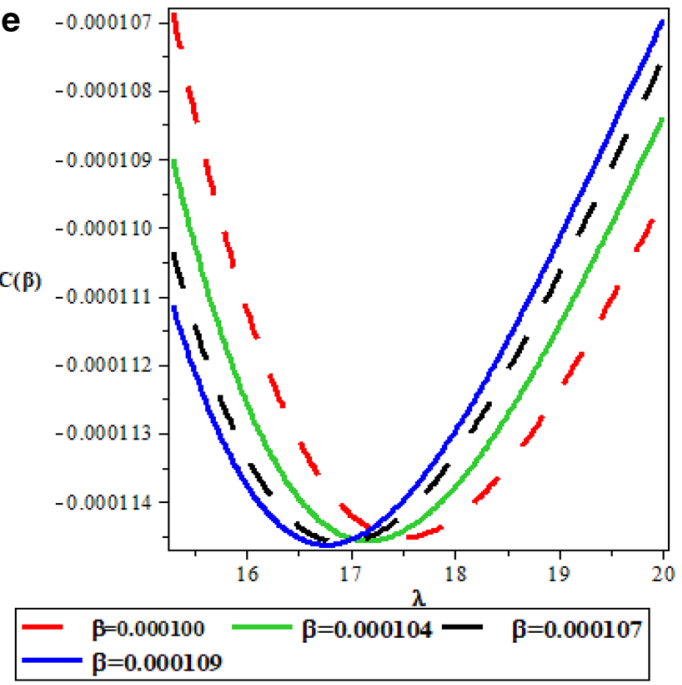

b

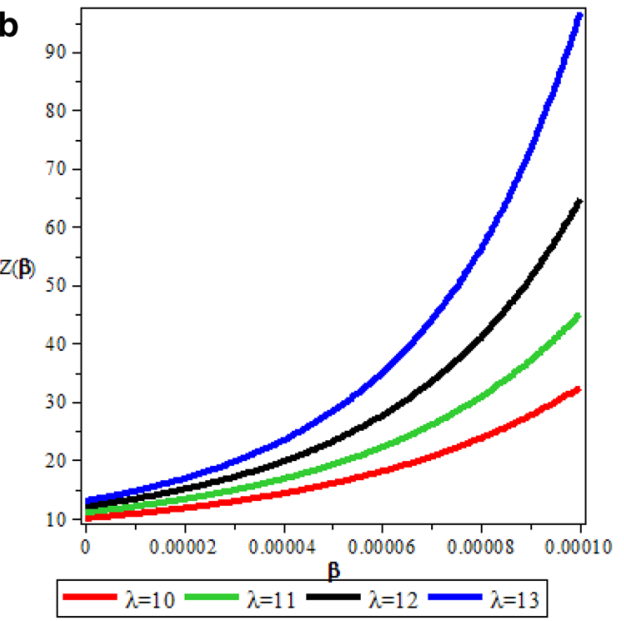

d

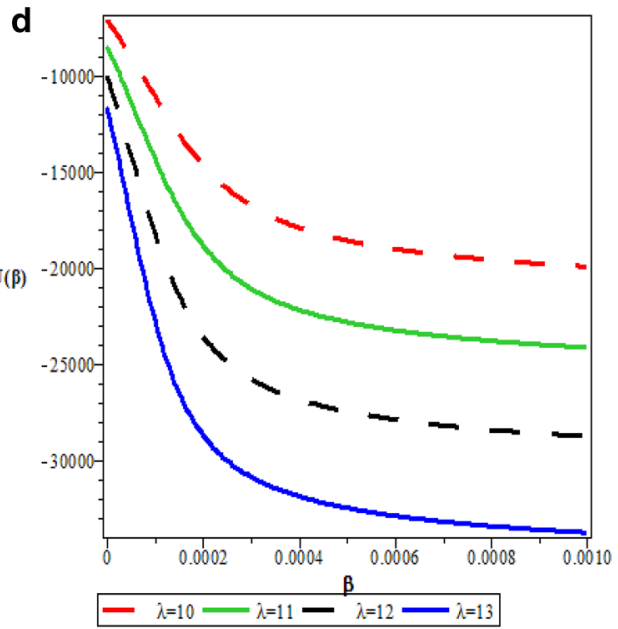

$\mathbf{f}$

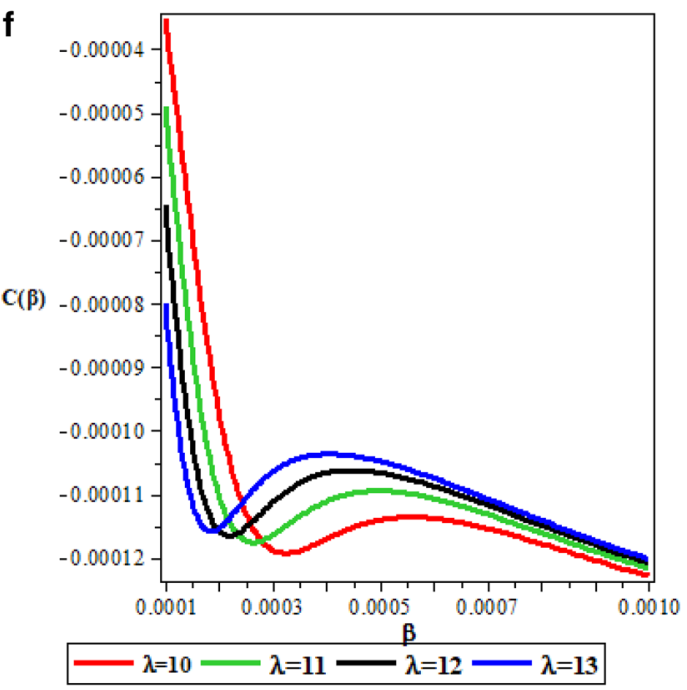

Figure 2. (a) Variation of partition function with respect to $\lambda$. (b) Variation of partition function with respect to $\beta$. (c) Variation of vibrational mean energy with respect to $\lambda$. (d) Variation of vibrational mean energy with respect to $\beta$. (e) Variation of specific heat capacity with respect to $\lambda$. (f) Variation of specific heat capacity with respect to $\beta$. (g) Variation of vibrational entropy with respect to $\lambda$. (h) Variation of vibrational entropy with respect to $\beta$. (i) Variation of vibrational free energy with respect to $\lambda$. (j) Variation of vibrational free energy with respect to $\beta$. 

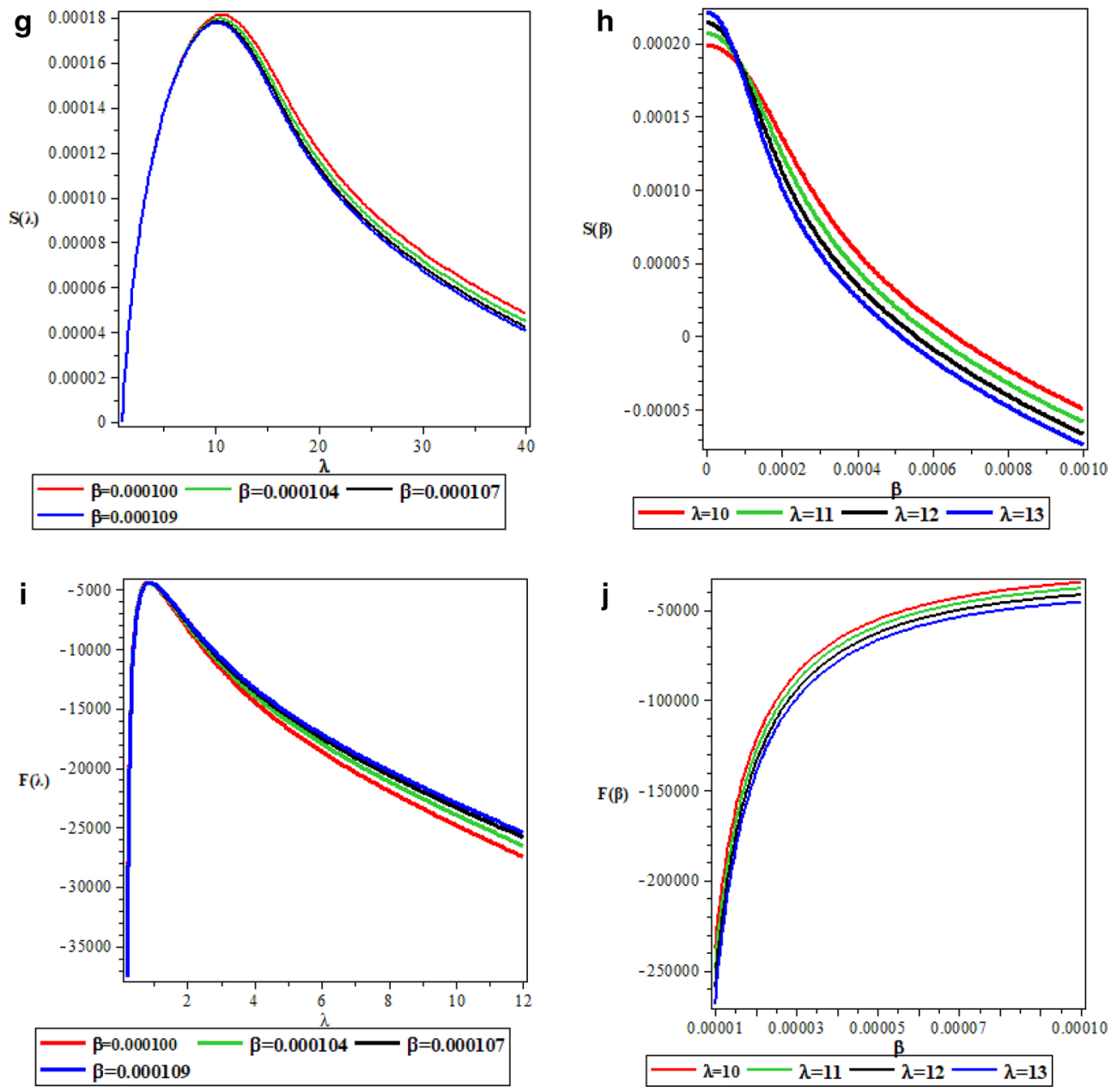

Figure 2. (continued)

\begin{tabular}{|l|c|l|l|l|l|l|l|l|}
\hline $\boldsymbol{\ell}$ & $\boldsymbol{k}<\mathbf{0}$ & $\boldsymbol{n}$ & $(\boldsymbol{\ell}, \boldsymbol{j})$ & $\boldsymbol{E}_{\boldsymbol{n} \boldsymbol{k}}$ & $\boldsymbol{k}>\mathbf{0}$ & $\boldsymbol{n}$ & $(\boldsymbol{\ell}, \boldsymbol{j})$ & $\boldsymbol{E}_{\boldsymbol{n} \boldsymbol{k}}$ \\
\hline 0 & -1 & 0 & $0 s_{\frac{1}{2}}$ & 8.983921278 & 1 & 0 & $0 s_{\frac{1}{2}}$ & -8.223233399 \\
\hline 0 & -1 & 1 & $1 s_{\frac{1}{2}}$ & 3.255885745 & 1 & 1 & $1 s_{\frac{1}{2}}$ & 2.349810532 \\
\hline 0 & -1 & 2 & $2 s_{\frac{1}{2}}$ & 3.764133532 & 1 & 2 & $2 s_{\frac{1}{2}}$ & 3.244054374 \\
\hline 0 & -1 & 3 & $3 s_{\frac{1}{2}}$ & 4.671917081 & 1 & 3 & $3 s_{\frac{1}{2}}$ & 4.275589604 \\
\hline 1 & -2 & 0 & $0 p_{\frac{3}{2}}$ & -8.223233399 & 2 & 0 & $0 p_{\frac{3}{2}}$ & -7.512110433 \\
\hline 1 & -2 & 1 & $1 p_{\frac{3}{2}}$ & 2.349810532 & 2 & 1 & $1 p_{\frac{3}{2}}$ & 0.6388962715 \\
\hline 1 & -2 & 2 & $2 p_{\frac{3}{2}}$ & 3.244054374 & 2 & 2 & $2 p_{\frac{3}{2}}$ & 2.246255163 \\
\hline 1 & -2 & 3 & $3 p_{\frac{3}{2}}$ & 4.275589604 & 2 & 3 & $3 p_{\frac{3}{2}}$ & 3.505707617 \\
\hline 2 & -3 & 0 & $0 d_{\frac{5}{2}}$ & -7.512110433 & 3 & 0 & $0 d_{\frac{5}{2}}$ & -7.240908451 \\
\hline 2 & -3 & 1 & $1 d_{\frac{5}{2}}$ & 0.6388962715 & 3 & 1 & $1 d_{\frac{5}{2}}$ & -1.407169997 \\
\hline 2 & -3 & 2 & $2 d_{\frac{5}{2}}$ & 2.246255163 & 3 & 2 & $2 d_{\frac{5}{2}}$ & 0.8693835435 \\
\hline 2 & -3 & 3 & $3 d_{\frac{5}{2}}$ & 3.505707617 & 3 & 3 & $3 d_{\frac{5}{2}}$ & 2.410060646 \\
\hline 3 & -4 & 0 & $0 f_{\frac{7}{2}}$ & -7.240908451 & 4 & 0 & $0 f_{\frac{7}{2}}$ & -7.586751966 \\
\hline 3 & -4 & 1 & $1 f_{\frac{7}{2}}$ & -1.407169997 & 4 & 1 & $1 f_{\frac{7}{2}}$ & -3.30875422 \\
\hline 3 & -4 & 2 & $2 f_{\frac{7}{2}}$ & 0.8693835435 & 4 & 2 & $2 f_{\frac{7}{2}}$ & -0.7385660036 \\
\hline 3 & -4 & 3 & $3 f_{\frac{7}{2}}$ & 2.410060646 & 4 & 3 & $3 f_{\frac{7}{2}}$ & 1.060283723 \\
\hline
\end{tabular}

Table 1. Numerical values for spin symmetry energies for $A=5 \mathrm{fm}^{-1}, v_{1}=15 \mathrm{fm}^{-1}, b=0.1, M=10 \mathrm{fm}^{-1},(\hbar=c=1)$ unit. 


\begin{tabular}{|l|l|l|l|l|l|l|l|r|}
\hline $\boldsymbol{\ell}$ & $\boldsymbol{k}<\mathbf{0}$ & $\boldsymbol{n}$ & $(\boldsymbol{\ell}, \boldsymbol{j})$ & $\boldsymbol{E}_{\boldsymbol{n} \boldsymbol{k}}$ & $\boldsymbol{k} \boldsymbol{> 0}$ & $\boldsymbol{n}$ & $(\boldsymbol{\ell}, \boldsymbol{j})$ & $\boldsymbol{E}_{\boldsymbol{n} \boldsymbol{k}}$ \\
\hline 0 & -1 & 0 & $0 s_{\frac{1}{2}}$ & 10.65566240 & 1 & 0 & $0 s_{\frac{1}{2}}$ & -9.402539935 \\
\hline 0 & -1 & 1 & $1 s_{\frac{1}{2}}$ & 8.879147410 & 1 & 1 & $1 s_{\frac{1}{2}}$ & 8.593038708 \\
\hline 0 & -1 & 2 & $2 s_{\frac{1}{2}}$ & 6.851894743 & 1 & 2 & $2 s_{\frac{1}{2}}$ & 6.412449813 \\
\hline 0 & -1 & 3 & $3 s_{\frac{1}{2}}$ & 6.591830427 & 1 & 3 & $3 s_{\frac{1}{2}}$ & 6.277759731 \\
\hline 1 & -2 & 0 & $0 p_{\frac{3}{2}}$ & -9.402539935 & 2 & 0 & $0 p_{\frac{3}{2}}$ & -8.862687863 \\
\hline 1 & -2 & 1 & $1 p_{\frac{3}{2}}$ & 8.593038708 & 2 & 1 & $1 p_{\frac{3}{2}}$ & 8.010408756 \\
\hline 1 & -2 & 2 & $2 p_{\frac{3}{2}}$ & 6.412449813 & 2 & 2 & $2 p_{\frac{3}{2}}$ & 5.467061490 \\
\hline 1 & -2 & 3 & $3 p_{\frac{3}{2}}$ & 6.257759731 & 2 & 3 & $3 p_{\frac{3}{2}}$ & 5.589744715 \\
\hline 2 & -3 & 0 & $0 d_{\frac{5}{2}}$ & -8.862687863 & 3 & 0 & $0 d_{\frac{5}{2}}$ & -8.201366952 \\
\hline 2 & -3 & 1 & $1 d_{\frac{5}{2}}$ & 8.010408756 & 3 & 1 & $1 d_{\frac{5}{2}}$ & -5.105451949 \\
\hline 2 & -3 & 2 & $2 d_{\frac{5}{2}}$ & 5.467061490 & 3 & 2 & $2 d_{\frac{5}{2}}$ & 3.898663797 \\
\hline 2 & -3 & 3 & $3 d_{\frac{5}{2}}$ & 5.589744715 & 3 & 3 & $3 d_{\frac{5}{2}}$ & 4.606019730 \\
\hline 3 & -4 & 0 & $0 f_{\frac{7}{2}}$ & -8.201366952 & 4 & 0 & $0 f_{\frac{7}{2}}$ & -7.510682389 \\
\hline 3 & -4 & 1 & $1 f_{\frac{7}{2}}$ & -5.105451949 & 4 & 1 & $1 f_{\frac{7}{2}}$ & -3.748571939 \\
\hline 3 & -4 & 2 & $2 f_{\frac{7}{2}}$ & 3.898663797 & 4 & 2 & $2 f_{\frac{7}{2}}$ & 1.865629960 \\
\hline 3 & -4 & 3 & $3 f_{\frac{7}{2}}$ & 4.606019730 & 4 & 3 & $3 f_{\frac{7}{2}}$ & 3.379690499 \\
\hline
\end{tabular}

Table 2. Numerical values for spin symmetry energies for $A=5 \mathrm{fm}^{-1}, v_{1}=15 \mathrm{fm}^{-1}, b=0.2, M=10 \mathrm{fm}^{-1},(\hbar=c=1)$ unit.

\begin{tabular}{|l|l|l|l|l|l|l|l|l|}
\hline $\boldsymbol{\ell}$ & $\boldsymbol{k}<\mathbf{0}$ & $\boldsymbol{n}$ & $(\boldsymbol{\ell}, \boldsymbol{j})$ & $\boldsymbol{E}_{\boldsymbol{n} \boldsymbol{k}}$ & $\boldsymbol{k}>\mathbf{0}$ & $\boldsymbol{n}$ & $(\boldsymbol{\ell}, \boldsymbol{j})$ & $\boldsymbol{E}_{\boldsymbol{n} \boldsymbol{k}}$ \\
\hline 0 & -1 & 0 & $0 s_{\frac{1}{2}}$ & 11.94562892 & 1 & 0 & $0 s_{\frac{1}{2}}$ & -9.653054948 \\
\hline 0 & -1 & 1 & $1 s_{\frac{1}{2}}$ & 11.45354051 & 1 & 1 & $1 s_{\frac{1}{2}}$ & 11.30816398 \\
\hline 0 & -1 & 2 & $2 s_{\frac{1}{2}}$ & 10.64354064 & 1 & 2 & $2 s_{\frac{1}{2}}$ & 10.45259601 \\
\hline 0 & -1 & 3 & $3 s_{\frac{1}{2}}$ & 9.931274480 & 1 & 3 & $3 s_{\frac{1}{2}}$ & 9.710639231 \\
\hline 1 & -2 & 0 & $0 p_{\frac{3}{2}}$ & -9.653054948 & 2 & 0 & $0 p_{\frac{3}{2}}$ & -9.226504128 \\
\hline 1 & -2 & 1 & $1 p_{\frac{3}{2}}$ & 11.30816398 & 2 & 1 & $1 p_{\frac{3}{2}}$ & 11.01670360 \\
\hline 1 & -2 & 2 & $2 p_{\frac{3}{2}}$ & 10.45259601 & 2 & 2 & $2 p_{\frac{3}{2}}$ & 10.05799030 \\
\hline 1 & -2 & 3 & $3 p_{\frac{3}{2}}$ & 9.710639231 & 2 & 3 & $3 p_{\frac{3}{2}}$ & 9.251447327 \\
\hline 2 & -3 & 0 & $0 d_{\frac{5}{2}}$ & -9.226504128 & 3 & 0 & $0 d_{\frac{5}{2}}$ & -8.642597290 \\
\hline 2 & -3 & 1 & $1 d_{\frac{5}{2}}$ & 11.01670360 & 3 & 1 & $1 d_{\frac{5}{2}}$ & -7.704698788 \\
\hline 2 & -3 & 2 & $2 d_{\frac{5}{2}}$ & 10.05799030 & 3 & 2 & $2 d_{\frac{5}{2}}$ & 9.428222506 \\
\hline 2 & -3 & 3 & $3 d_{\frac{5}{2}}$ & 9.251447327 & 3 & 3 & $3 d_{\frac{5}{2}}$ & 8.510496320 \\
\hline 3 & -4 & 0 & $0 f_{\frac{7}{2}}$ & -8.642597290 & 4 & 0 & $0 f_{\frac{7}{2}}$ & -7.911699446 \\
\hline 3 & -4 & 1 & $1 f_{\frac{7}{2}}$ & -7.704698788 & 4 & 1 & $1 f_{\frac{7}{2}}$ & -6.620201873 \\
\hline 3 & -4 & 2 & $2 f_{\frac{7}{2}}$ & 9.428222506 & 4 & 2 & $2 f_{\frac{7}{2}}$ & 8.493229223 \\
\hline 3 & -4 & 3 & $3 f_{\frac{7}{2}}$ & 8.510496320 & 4 & 3 & $3 f_{\frac{7}{2}}$ & 7.400643640 \\
\hline
\end{tabular}

Table 3. Numerical values for spin symmetry energies for $A=5 \mathrm{fm}^{-1}, v_{1}=15 \mathrm{fm}^{-1}, b=0.3, M=10 \mathrm{fm}^{-1},(\hbar=c=1)$ unit. 


\begin{tabular}{|l|l|l|l|l|l|l|l|l|}
\hline$\ell$ & $\boldsymbol{k}<\mathbf{0}$ & $\boldsymbol{n}$ & $(\boldsymbol{\ell}, \boldsymbol{j})$ & $\boldsymbol{E}_{\boldsymbol{n} \boldsymbol{k}}$ & $\boldsymbol{k}>\mathbf{0}$ & $\boldsymbol{n}$ & $(\boldsymbol{\ell}, \boldsymbol{j})$ & $\boldsymbol{E}_{\boldsymbol{n} \boldsymbol{k}}$ \\
\hline 0 & -1 & 0 & $0 s_{\frac{1}{2}}$ & 13.56961400 & 1 & 0 & $0 s_{\frac{1}{2}}$ & -9.759490473 \\
\hline 0 & -1 & 1 & $1 s_{\frac{1}{2}}$ & 13.42954857 & 1 & 1 & $1 s_{\frac{1}{2}}$ & 13.32679497 \\
\hline 0 & -1 & 2 & $2 s_{\frac{1}{2}}$ & 13.18355639 & 1 & 2 & $2 s_{\frac{1}{2}}$ & 13.7149132 \\
\hline 0 & -1 & 3 & $3 s_{\frac{1}{2}}$ & 12.89651062 & 1 & 3 & $3 s_{\frac{1}{2}}$ & 12.77434725 \\
\hline 1 & -2 & 0 & $0 p_{\frac{3}{2}}$ & -9.759490473 & 2 & 0 & $0 p_{\frac{3}{2}}$ & -9.405058745 \\
\hline 1 & -2 & 1 & $1 p_{\frac{3}{2}}$ & 13.32679497 & 2 & 1 & $1 p_{\frac{3}{2}}$ & -9.212559444 \\
\hline 1 & -2 & 2 & $2 p_{\frac{3}{2}}$ & 13.07149132 & 2 & 2 & $2 p_{\frac{3}{2}}$ & 12.84444065 \\
\hline 1 & -2 & 3 & $3 p_{\frac{3}{2}}$ & 12.77434725 & 2 & 3 & $3 p_{\frac{3}{2}}$ & 12.52547716 \\
\hline 2 & -3 & 0 & $0 d_{\frac{5}{2}}$ & -9.405058745 & 3 & 0 & $0 d_{\frac{5}{2}}$ & -8.898795585 \\
\hline 2 & -3 & 1 & $1 d_{\frac{5}{2}}$ & -9.212559444 & 3 & 1 & $1 d_{\frac{5}{2}}$ & -8.562364622 \\
\hline 2 & -3 & 2 & $2 d_{\frac{5}{2}}$ & 12.8444065 & 3 & 2 & $2 d_{\frac{5}{2}}$ & 12.49608256 \\
\hline 2 & -3 & 3 & $3 d_{\frac{5}{2}}$ & 12.52547716 & 3 & 3 & $3 d_{\frac{5}{2}}$ & 12.13969691 \\
\hline 3 & -4 & 0 & $0 f_{\frac{7}{2}}$ & -8.898795585 & 4 & 0 & $0 f_{\frac{7}{2}}$ & -8.238455058 \\
\hline 3 & -4 & 1 & $1 f_{\frac{7}{2}}$ & -8.562364622 & 4 & 1 & $1 f_{\frac{7}{2}}$ & -7.749637734 \\
\hline 3 & -4 & 2 & $2 f_{\frac{7}{2}}$ & 12.49608256 & 4 & 2 & $2 f_{\frac{7}{2}}$ & -7.377835097 \\
\hline 3 & -4 & 3 & $3 f_{\frac{7}{2}}$ & 12.1396969 & 4 & 3 & $3 f_{\frac{7}{2}}$ & 11.59818143 \\
\hline
\end{tabular}

Table 4. Numerical values for spin symmetry energies for $A=5 \mathrm{fm}^{-1}, v_{1}=15 \mathrm{fm}^{-1}, b=0.4, M=10 \mathrm{fm}^{-1},(\hbar=c=1)$ unit.

\begin{tabular}{|l|l|l|l|l|l|l|l|l|}
\hline $\boldsymbol{\ell}$ & $\boldsymbol{k}<\mathbf{0}$ & $\boldsymbol{n}$ & $(\boldsymbol{\ell}, \boldsymbol{j})$ & $\boldsymbol{E}_{\boldsymbol{n} \boldsymbol{k}}$ & $\boldsymbol{k}>\mathbf{0}$ & $\boldsymbol{n}$ & $(\boldsymbol{\ell}, \boldsymbol{j})$ & $\boldsymbol{E}_{\boldsymbol{n} \boldsymbol{k}}$ \\
\hline 0 & -1 & 0 & $0 s_{\frac{1}{2}}$ & -12.69213560 & 1 & 0 & $0 s_{\frac{1}{2}}$ & -12.30271714 \\
\hline 0 & -1 & 1 & $1 s_{\frac{1}{2}}$ & -20.05606687 & 1 & 1 & $1 s_{\frac{1}{2}}$ & -19.58322167 \\
\hline 0 & -1 & 2 & $2 s_{\frac{1}{2}}$ & -28.51380132 & 1 & 2 & $2 s_{\frac{1}{2}}$ & -28.07267520 \\
\hline 0 & -1 & 3 & $3 s_{\frac{1}{2}}$ & -37.19205174 & 1 & 3 & $3 s_{\frac{1}{2}}$ & -36.79032895 \\
\hline 1 & -2 & 0 & $0 p_{\frac{3}{2}}$ & -13.50050752 & 2 & 0 & $0 p_{\frac{3}{2}}$ & -12.69213560 \\
\hline 1 & -2 & 1 & $1 p_{\frac{3}{2}}$ & -20.98411765 & 2 & 1 & $1 p_{\frac{3}{2}}$ & -20.05606687 \\
\hline 1 & -2 & 2 & $2 p_{\frac{3}{2}}$ & -29.37941053 & 2 & 2 & $2 p_{\frac{3}{2}}$ & -28.51380132 \\
\hline 1 & -2 & 3 & $3 p_{\frac{3}{2}}$ & -37.98303228 & 2 & 3 & $3 p_{\frac{3}{2}}$ & -37.19205174 \\
\hline 2 & -3 & 0 & $0 d_{\frac{5}{2}}$ & -14.75172391 & 3 & 0 & $0 d_{\frac{5}{2}}$ & -13.50050752 \\
\hline 2 & -3 & 1 & $1 d_{\frac{5}{2}}$ & -22.33384979 & 3 & 1 & $1 d_{\frac{5}{2}}$ & -20.98411765 \\
\hline 2 & -3 & 2 & $2 d_{\frac{5}{2}}$ & -30.63932420 & 3 & 2 & $2 d_{\frac{5}{2}}$ & -29.37941053 \\
\hline 2 & -3 & 3 & $3 d_{\frac{5}{2}}$ & -39.14034408 & 3 & 3 & $3 d_{\frac{5}{2}}$ & -37.98303228 \\
\hline 3 & -4 & 0 & $0 f_{\frac{7}{2}}$ & -16.43288886 & 4 & 0 & $0 f_{\frac{7}{2}}$ & -14.75172391 \\
\hline 3 & -4 & 1 & $1 f_{\frac{7}{2}}$ & -24.06031886 & 4 & 1 & $1 f_{\frac{7}{2}}$ & -22.33384979 \\
\hline 3 & -4 & 2 & $2 f_{\frac{7}{2}}$ & -32.25483876 & 4 & 2 & $2 f_{\frac{7}{2}}$ & -30.63932420 \\
\hline 3 & -4 & 3 & $3 f_{\frac{7}{2}}$ & -40.63390244 & 4 & 3 & $3 f_{\frac{7}{2}}$ & -39.14034408 \\
\hline
\end{tabular}

Table 5. Numerical values for pseudospin symmetry energies for $A=5 \mathrm{fm}^{-1}, v_{1}=15 \mathrm{fm}^{-1}, b=0.1$, $M=10 \mathrm{fm}^{-1},(\hbar=c=1)$ unit. 


\begin{tabular}{|l|l|l|l|l|l|l|l|l|}
\hline $\boldsymbol{\ell}$ & $\boldsymbol{k}<\mathbf{0}$ & $\boldsymbol{n}$ & $(\boldsymbol{\ell}, \boldsymbol{j})$ & $\boldsymbol{E}_{\boldsymbol{n} \boldsymbol{k}}$ & $\boldsymbol{k}>\mathbf{0}$ & $\boldsymbol{n}$ & $(\boldsymbol{\ell}, \boldsymbol{j})$ & $\boldsymbol{E}_{\boldsymbol{n} \boldsymbol{k}}$ \\
\hline 0 & -1 & 0 & $0 s_{\frac{1}{2}}$ & -12.28432355 & 1 & 0 & $0 s_{\frac{1}{2}}$ & -12.09991279 \\
\hline 0 & -1 & 1 & $1 s_{\frac{1}{2}}$ & -15.51562769 & 1 & 1 & $1 s_{\frac{1}{2}}$ & -15.29325472 \\
\hline 0 & -1 & 2 & $2 s_{\frac{1}{2}}$ & -19.25297714 & 1 & 2 & $2 s_{\frac{1}{2}}$ & -19.02772195 \\
\hline 0 & -1 & 3 & $3 s_{\frac{1}{2}}$ & -23.19071008 & 1 & 3 & $3 s_{\frac{1}{2}}$ & -22.97175688 \\
\hline 1 & -2 & 0 & $0 p_{\frac{3}{2}}$ & -12.66108726 & 2 & 0 & $0 p_{\frac{3}{2}}$ & -12.28432355 \\
\hline 1 & -2 & 1 & $1 p_{\frac{3}{2}}$ & -15.95750279 & 2 & 1 & $1 p_{\frac{3}{2}}$ & -15.51562769 \\
\hline 1 & -2 & 2 & $2 p_{\frac{3}{2}}$ & -19.70093833 & 2 & 2 & $2 p_{\frac{3}{2}}$ & -19.02772195 \\
\hline 1 & -2 & 3 & $3 p_{\frac{3}{2}}$ & -23.62302141 & 2 & 3 & $3 p_{\frac{3}{2}}$ & -23.19071008 \\
\hline 2 & -3 & 0 & $0 d_{\frac{5}{2}}$ & -13.24035926 & 3 & 0 & $0 d_{\frac{5}{2}}$ & -12.66108726 \\
\hline 2 & -3 & 1 & $1 d_{\frac{5}{2}}$ & -16.61218365 & 3 & 1 & $1 d_{\frac{5}{2}}$ & -15.95750279 \\
\hline 2 & -3 & 2 & $2 d_{\frac{5}{2}}$ & -20.35795593 & 3 & 2 & $2 d_{\frac{5}{2}}$ & -19.70093833 \\
\hline 2 & -3 & 3 & $3 d_{\frac{5}{2}}$ & -24.25815897 & 3 & 3 & $3 d_{\frac{5}{2}}$ & -23.62302141 \\
\hline 3 & -4 & 0 & $0 f_{\frac{7}{2}}$ & -14.02686986 & 4 & 0 & $0 f_{\frac{7}{2}}$ & -16.61218365 \\
\hline 3 & -4 & 1 & $1 f_{\frac{7}{2}}$ & -17.46826708 & 4 & 1 & $1 f_{\frac{7}{2}}$ & -16.61218365 \\
\hline 3 & -4 & 2 & $2 f_{\frac{7}{2}}$ & -21.21020722 & 4 & 2 & $2 f_{\frac{7}{2}}$ & -20.35795593 \\
\hline 3 & -4 & 3 & $3 f_{\frac{7}{2}}$ & -25.08181569 & 4 & 3 & $3 f_{\frac{7}{2}}$ & -24.25815897 \\
\hline
\end{tabular}

Table 6. Numerical values for pseudospin symmetry energies for $A=5 \mathrm{fm}^{-1}, v_{1}=15 \mathrm{fm}^{-1}, b=0.2, M=10 \mathrm{fm}^{-1},(\hbar=c=1)$ unit

\begin{tabular}{|l|l|l|l|l|l|l|l|l|}
\hline $\boldsymbol{\ell}$ & $\boldsymbol{k}<\mathbf{0}$ & $\boldsymbol{n}$ & $(\boldsymbol{\ell}, \boldsymbol{j})$ & $\boldsymbol{E}_{\boldsymbol{n} \boldsymbol{k}}$ & $\boldsymbol{k}>\mathbf{0}$ & $\boldsymbol{n}$ & $(\boldsymbol{\ell}, \boldsymbol{j})$ & $\boldsymbol{E}_{\boldsymbol{n} \boldsymbol{k}}$ \\
\hline 0 & -1 & 0 & $0 s_{\frac{1}{2}}$ & -13.10449580 & 1 & 0 & $0 s_{\frac{1}{2}}$ & -12.97416326 \\
\hline 0 & -1 & 1 & $1 s_{\frac{1}{2}}$ & -15.24478036 & 1 & 1 & $1 s_{\frac{1}{2}}$ & -15.10293877 \\
\hline 0 & -1 & 2 & $2 s_{\frac{1}{2}}$ & -17.57946118 & 1 & 2 & $2 s_{\frac{1}{2}}$ & -17.43537205 \\
\hline 0 & -1 & 3 & $3 s_{\frac{1}{2}}$ & -20.01181246 & 1 & 3 & $3 s_{\frac{1}{2}}$ & -19.86975971 \\
\hline 1 & -2 & 0 & $0 p_{\frac{3}{2}}$ & -13.365886250 & 2 & 0 & $0 p_{\frac{3}{2}}$ & -13.10449580 \\
\hline 1 & -2 & 1 & $1 p_{\frac{3}{2}}$ & -15.52663385 & 2 & 1 & $1 p_{\frac{3}{2}}$ & -15.24478036 \\
\hline 1 & -2 & 2 & $2 p_{\frac{3}{2}}$ & -17.86488937 & 2 & 2 & $2 p_{\frac{3}{2}}$ & -17.57946118 \\
\hline 1 & -2 & 3 & $3 p_{\frac{3}{2}}$ & -20.29298159 & 2 & 3 & $3 p_{\frac{3}{2}}$ & -20.01181246 \\
\hline 2 & -3 & 0 & $0 d_{\frac{5}{2}}$ & -13.75860256 & 3 & 0 & $0 d_{\frac{5}{2}}$ & -17.57946118 \\
\hline 2 & -3 & 1 & $1 d_{\frac{5}{2}}$ & -15.94464389 & 3 & 1 & $1 d_{\frac{5}{2}}$ & -1.552663385 \\
\hline 2 & -3 & 2 & $2 d_{\frac{5}{2}}$ & -18.28631249 & 3 & 2 & $2 d_{\frac{5}{2}}$ & -17.86488937 \\
\hline 2 & -3 & 3 & $3 d_{\frac{5}{2}}$ & -20.70765586 & 3 & 3 & $3 d_{\frac{5}{2}}$ & -20.29298159 \\
\hline 3 & -4 & 0 & $0 f_{\frac{7}{2}}$ & -14.28119674 & 4 & 0 & $0 f_{\frac{7}{2}}$ & -13.75860256 \\
\hline 3 & -4 & 1 & $1 f_{\frac{7}{2}}$ & -16.49274719 & 4 & 1 & $1 f_{\frac{7}{2}}$ & -15.94464389 \\
\hline 3 & -4 & 2 & $2 f_{\frac{7}{2}}$ & -18.83612438 & 4 & 2 & $2 f_{\frac{7}{2}}$ & -18.28631249 \\
\hline 3 & -4 & 3 & $3 f_{\frac{7}{2}}$ & -21.24802969 & 4 & 3 & $3 f_{\frac{7}{2}}$ & -20.70765586 \\
\hline
\end{tabular}

Table 7. Numerical values for pseudospin symmetry energies for $A=5 \mathrm{fm}^{-1}, v_{1}=15 \mathrm{fm}^{-1}, b=0.3, M=10 \mathrm{fm} \mathrm{m}^{-1}$, $(\hbar=c=1)$ unit. 


\begin{tabular}{|l|l|l|l|l|l|l|l|l|}
\hline $\boldsymbol{\ell}$ & $\boldsymbol{k}<\mathbf{0}$ & $\boldsymbol{n}$ & $(\boldsymbol{\ell}, \boldsymbol{j})$ & $\boldsymbol{E}_{\boldsymbol{n} \boldsymbol{k}}$ & $\boldsymbol{k}>\mathbf{0}$ & $\boldsymbol{n}$ & $(\boldsymbol{\ell}, \boldsymbol{j})$ & $\boldsymbol{E}_{\boldsymbol{n} \boldsymbol{k}}$ \\
\hline 0 & -1 & 0 & $0 s_{\frac{1}{2}}$ & -14.47035819 & 1 & 0 & $0 s_{\frac{1}{2}}$ & -14.37133775 \\
\hline 0 & -1 & 1 & $1 s_{\frac{1}{2}}$ & -16.06990567 & 1 & 1 & $1 s_{\frac{1}{2}}$ & -15.96860262 \\
\hline 0 & -1 & 2 & $2 s_{\frac{1}{2}}$ & -17.735611113 & 1 & 2 & $2 s_{\frac{1}{2}}$ & -17.63433738 \\
\hline 0 & -1 & 3 & $3 s_{\frac{1}{2}}$ & -19.43972082 & 1 & 3 & $3 s_{\frac{1}{2}}$ & -19.33973124 \\
\hline 1 & -2 & 0 & $0 p_{\frac{3}{2}}$ & -14.66732944 & 2 & 0 & $0 p_{\frac{3}{2}}$ & -14.47035819 \\
\hline 1 & -2 & 1 & $1 p_{\frac{3}{2}}$ & -16.27093685 & 2 & 1 & $1 p_{\frac{3}{2}}$ & -16.06990567 \\
\hline 1 & -2 & 2 & $2 p_{\frac{3}{2}}$ & -17.93639247 & 2 & 2 & $2 p_{\frac{3}{2}}$ & -17.73561113 \\
\hline 1 & -2 & 3 & $3 p_{\frac{3}{2}}$ & -19.63790665 & 2 & 3 & $3 p_{\frac{3}{2}}$ & -19.43972082 \\
\hline 2 & -3 & 0 & $0 d_{\frac{5}{2}}$ & -14.96002329 & 3 & 0 & $0 d_{\frac{5}{2}}$ & -14.66732944 \\
\hline 2 & -3 & 1 & $1 d_{\frac{5}{2}}$ & -16.56860653 & 3 & 1 & $1 d_{\frac{5}{2}}$ & -16.27093685 \\
\hline 2 & -3 & 2 & $2 d_{\frac{5}{2}}$ & -18.23327378 & 3 & 2 & $2 d_{\frac{5}{2}}$ & -17.93639247 \\
\hline 2 & -3 & 3 & $3 d_{\frac{5}{2}}$ & -19.93084754 & 3 & 3 & $3 d_{\frac{5}{2}}$ & -19.63790665 \\
\hline 3 & -4 & 0 & $0 f_{\frac{7}{2}}$ & -15.34495615 & 4 & 0 & $0 f_{\frac{7}{2}}$ & -14.96002329 \\
\hline 3 & -4 & 1 & $1 f_{\frac{7}{2}}$ & -16.95844684 & 4 & 1 & $1 f_{\frac{7}{2}}$ & -16.56860653 \\
\hline 3 & -4 & 2 & $2 f_{\frac{7}{2}}$ & -18.62144186 & 4 & 2 & $2 f_{\frac{7}{2}}$ & -18.23327378 \\
\hline 3 & -4 & 3 & $3 f_{\frac{7}{2}}$ & -20.31372099 & 4 & 3 & $3 f_{\frac{7}{2}}$ & -19.93084754 \\
\hline & & & & & & \\
\hline
\end{tabular}

Table 8. Numerical values for pseudospin symmetry energies for $A=5 \mathrm{fm}^{-1}, v_{1}=15 \mathrm{fm}^{-1}, b=0.4, M=10 \mathrm{fm}^{-1}$, $(\hbar=c=1)$ unit.

Finally, using Eq. (38) the numerical bound state solution is obtain for Hulthen potential as shown in Table 10. The numerical solutions obtained for Hulthen potential are in excellent agreement with Refs ${ }^{73-77}$.

\section{Conclusion}

In this research work, we obtained analytical solutions of Dirac equation for spin and pseudospin symmetries with hyperbolic Hulthen plus hyperbolic exponential inversely quadratic potential within the framework of parametric Nikiforov-Uvarov method. Using Maple software package, numerical solutions for both the spin and pseudospin symmetries were obtain. The nonrelativistic energy equation were obtain by applying nonrelativistic limit to the relativistic energy equation. The Partition function and other thermodynamic properties were obtain using the nonrelativistic energy equation presented in a close form. The proposed potential reduces to Hulthen and exponential inversely quadratic potential as special cases. The numerical results obtained for Hulthen potential, spin and pseudospin bound state energies and thermodynamic plots are in agreement to the work of other researchers. 
a
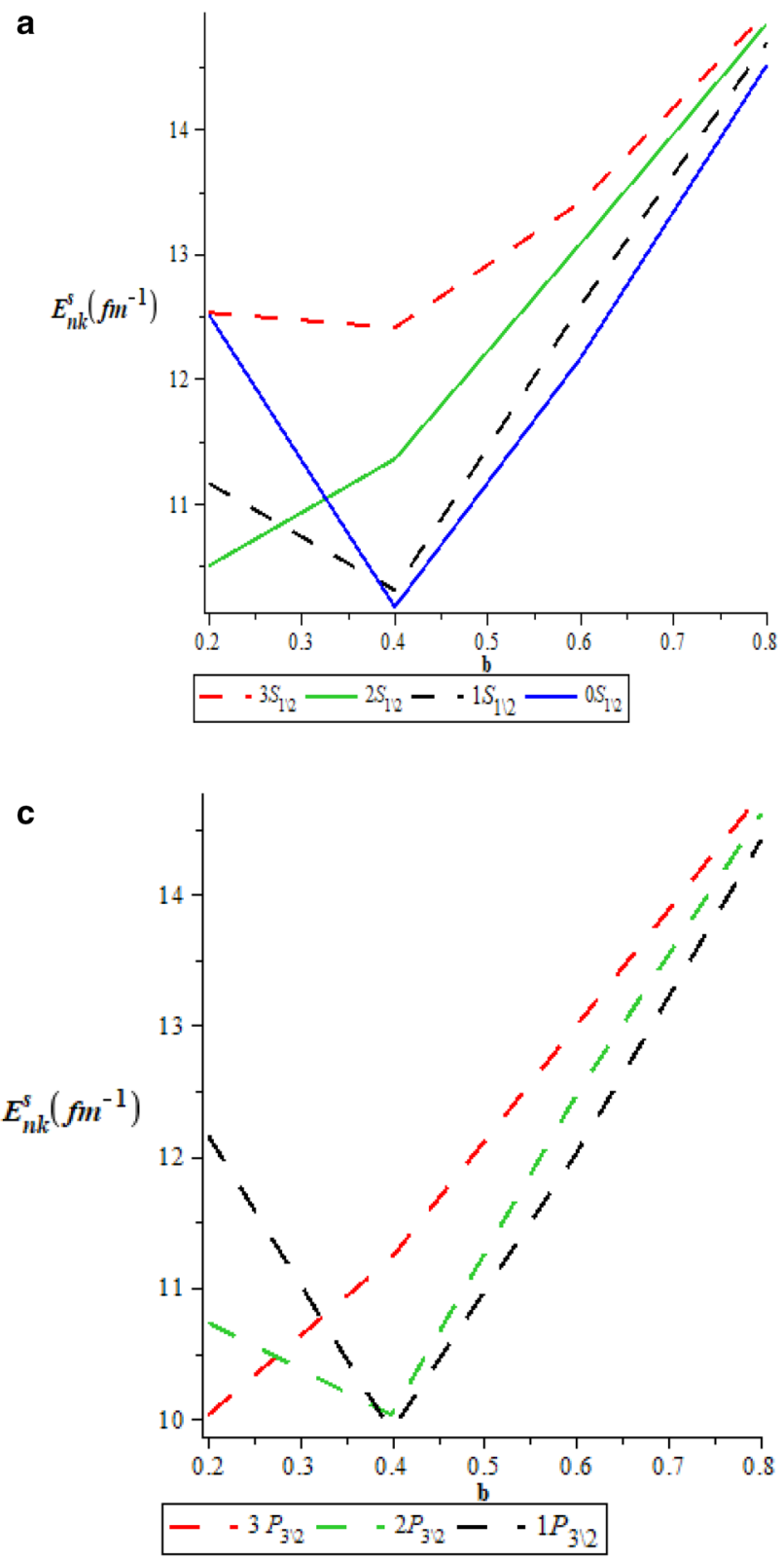

b

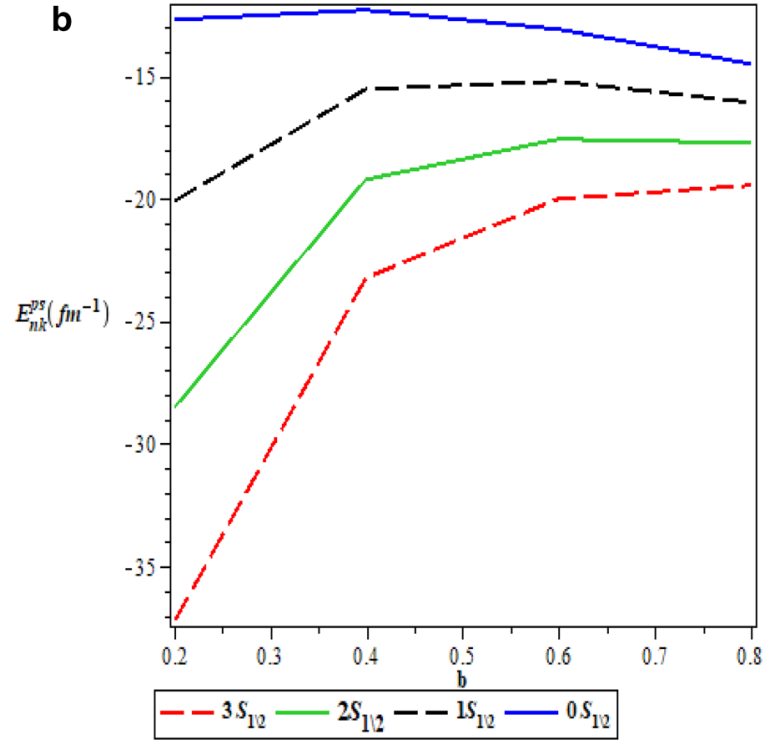

d

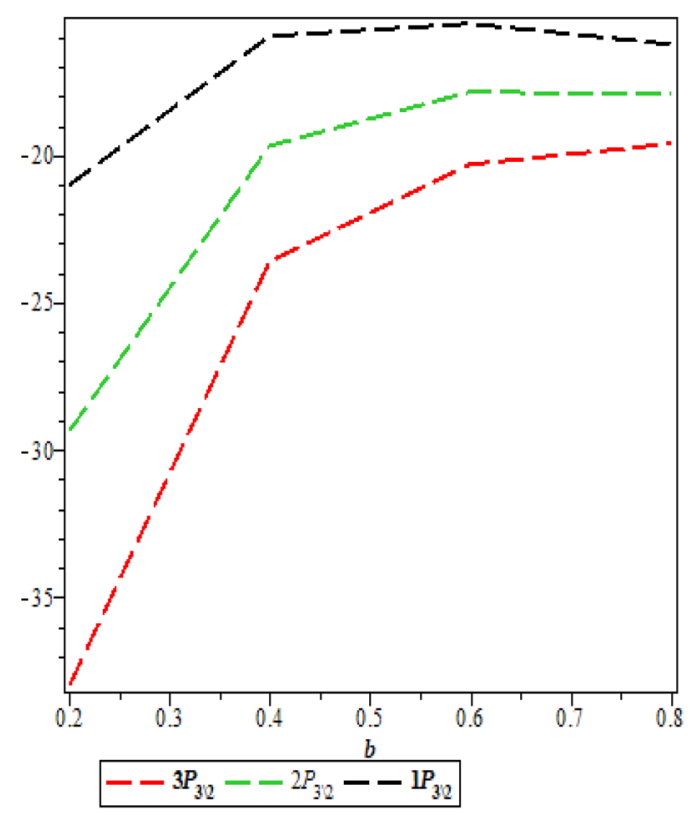

Figure 3. (a) Variation of Energy Spectra for S-spin Symmetry. (b) Variation of Energy Spectra for S-pseudospin Symmetry. (c) Variation of Energy Spectra for P-spin Symmetry. (d) Variation of Energy Spectra for P-pseudospin Symmetry. 
a

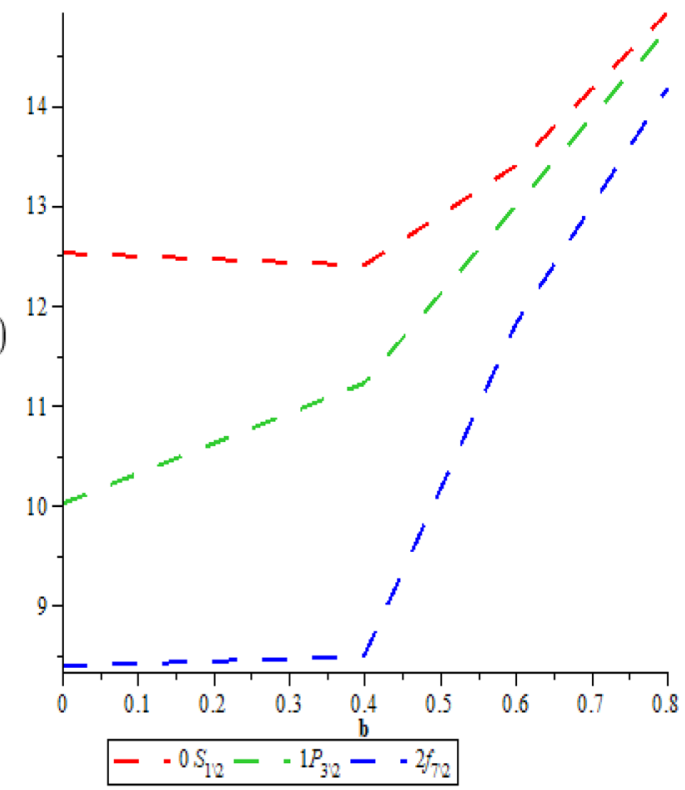

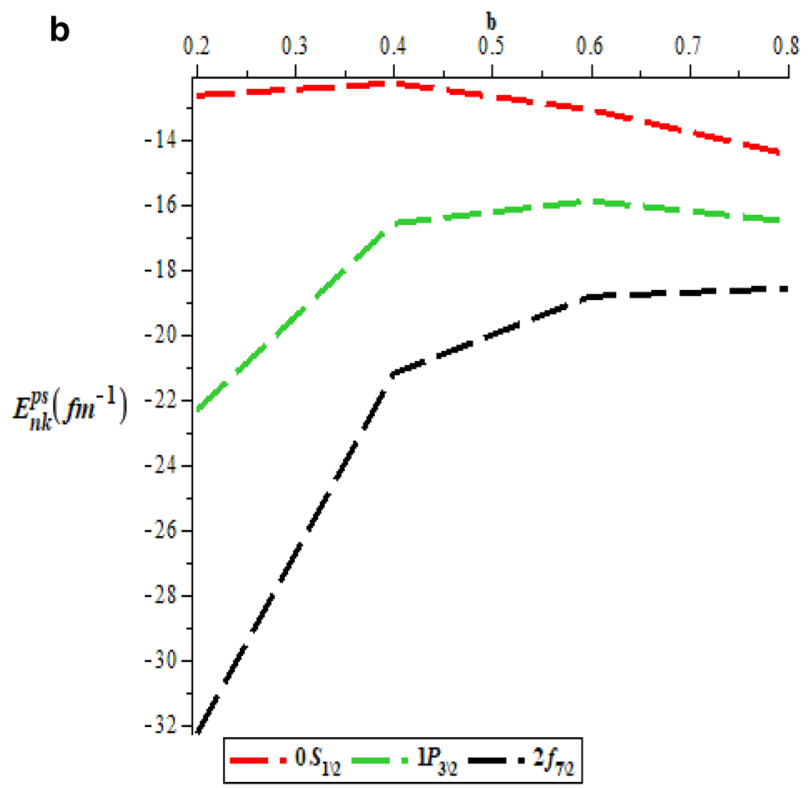

Figure 4. (a) Variation of Energy Spectra for Mixed Quantum spin. (b) Variation of Energy Spectra for Mixed Pseudo Quantum spin.

\begin{tabular}{|c|c|c|c|c|c|}
\hline$n$ & $l$ & $E_{n l}, b=0.1$ & $E_{n l}, b=0.2$ & $E_{n l}, b=0.3$ & $E_{n l}, b=0.4$ \\
\hline 0 & 0 & -12.0181250 & -2.3225000 & 0.520138889 & 1.30375000 \\
\hline \multirow{2}{*}{1} & 0 & -87.4934896 & -18.9583333 & -6.00622106 & -1.16536458 \\
\hline & 1 & -109.063125 & -24.5025000 & -8.56812500 & -2.66625000 \\
\hline \multirow{3}{*}{2} & 0 & -182.670166 & -40.7539062 & -14.4047580 & -5.10180664 \\
\hline & 1 & -214.749043 & -48.4604592 & -17.6280116 & -6.79145408 \\
\hline & 2 & -303.153125 & -68.8625000 & -25.6003472 & -10.606250 \\
\hline \multirow{4}{*}{3} & 0 & -279.82502 & -64.0625000 & -24.1323031 & -10.1872417 \\
\hline & 0 & -311.98140 & -71.7850000 & -27.3604340 & -11.8782812 \\
\hline & 0 & -602.045000 & -89.597656 & -34.5408257 & -15.4670410 \\
\hline & 0 & -279.82502 & -142.805000 & -57.9605556 & -28.5012500 \\
\hline \multirow{5}{*}{4} & 0 & -387.471458 & -90.6358333 & -35.7246065 & -16.5745833 \\
\hline & 1 & -417.501993 & -97.9209184 & -38.8189573 & -18.2274394 \\
\hline & 2 & -482.44108 & -113.49479 & -45.3146340 & -21.6207682 \\
\hline & 3 & -725.170139 & -174.222222 & -72.3337191 & -36.8368056 \\
\hline & 3 & -1003.75451 & -244.436412 & -103.906246 & -54.8202637 \\
\hline \multirow{6}{*}{5} & 0 & -513.819102 & -122.163125 & -49.6983030 & -24.4112891 \\
\hline & 1 & -542.076047 & -129.083276 & -52.6810413 & -26.0322888 \\
\hline & 2 & -600.711343 & -143.360078 & -58.7800185 & -29.3123919 \\
\hline & 3 & -879.002551 & -213.063776 & -89.8438209 & -46.8373724 \\
\hline & 4 & -1204.49792 & -294.812302 & -126.418037 & -67.5580482 \\
\hline & 5 & -12.0181250 & -369.920000 & -159.906806 & -86.4612500 \\
\hline
\end{tabular}

Table 9. Nonrelativistic numerical solutions for $A=5 \mathrm{fm}^{-1}, v_{1}=15 \mathrm{fm}^{-1}, b=0.1,(\hbar=c=1)$ unit. 

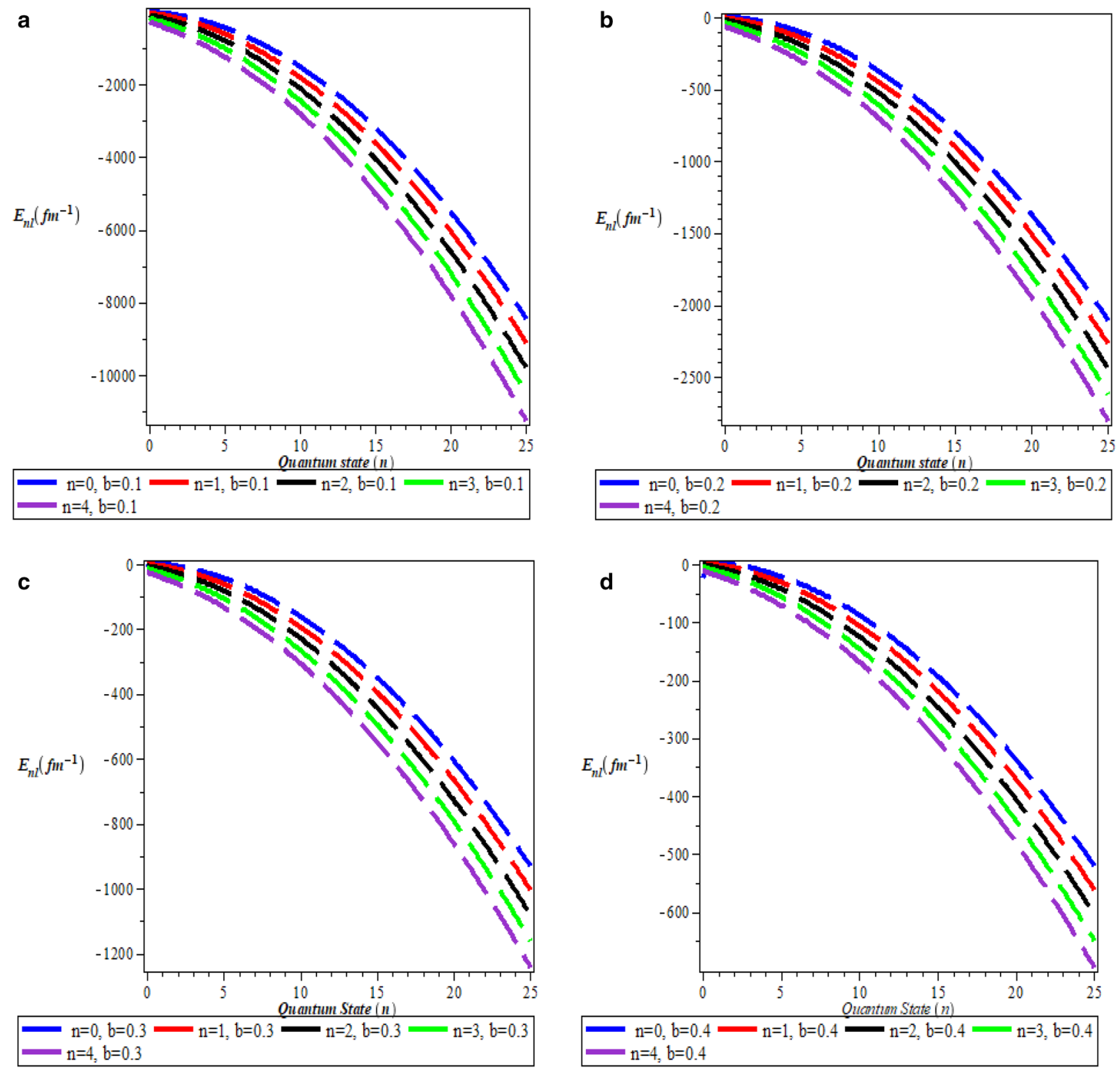

Figure 5. (a) Nonrelativistic energy spectra for $b=0.1$. (b) Nonrelativistic energy spectra for $b=0.2$. (c) Nonrelativistic energy spectra for $b=0.3$. (d) Nonrelativistic energy spectra for $b=0.4$. 


\begin{tabular}{|c|c|c|c|c|c|c|c|}
\hline State & $1 / b$ & Present & Jia et al. ${ }^{73}$ & Ikhdair $^{74}$ & Bayrak et al. ${ }^{75}$ & Varshni $^{{ }^{76}}$ & Stanek $^{77}$ \\
\hline \multirow{8}{*}{$2 p$} & 0.025 & 0.1128125 & 0.1126344 & 0.1127611 & 0.1127605 & 0.1127605 & 0.1127604 \\
\hline & 0.050 & 0.1012500 & 0.1009128 & 0.1010442 & 0.1010425 & 0.1010425 & 0.101042 \\
\hline & 0.075 & 0.0903120 & 0.0898350 & 0.0898495 & 0.0898478 & 0.0898478 & 0.0898453 \\
\hline & 0.100 & 0.0800000 & 0.0794011 & 0.0791769 & 0.0791794 & 0.0791794 & 0.0791717 \\
\hline & 0.150 & 0.0612500 & 0.0604650 & 0.0593981 & 0.0594415 & 0.0594415 & 0.0594007 \\
\hline & 0.200 & 0.0450000 & 0.0441045 & 0.0417078 & 0.0418861 & 0.0418860 & 0.0417491 \\
\hline & 0.250 & 0.0312500 & 0.0303195 & 0.0261059 & 0.0266111 & 0.0266111 & 0.0262466 \\
\hline & 0.300 & 0.0199994 & 0.0191101 & 0.0125925 & 0.0137900 & 0.0137900 & 0.0129347 \\
\hline \multirow{5}{*}{$3 p$} & 0.025 & 0.0437587 & 0.0436848 & 0.0437072 & 0.0437069 & 0.0437069 & 0.0437066 \\
\hline & 0.050 & 0.0333681 & 0.0332390 & 0.0331623 & 0.0331645 & 0.0331645 & 0.0331602 \\
\hline & 0.075 & 0.0243837 & 0.0242183 & 0.0239207 & 0.0239397 & 0.0239397 & 0.0239173 \\
\hline & 0.100 & 0.0168056 & 0.0166227 & 0.0159825 & 0.0160537 & 0.0160537 & 0.0159798 \\
\hline & 0.150 & 0.0058680 & 0.0057067 & 0.0040162 & 0.004463 & 0.0044663 & 0.0040316 \\
\hline \multirow{4}{*}{$3 d$} & 0.025 & 0.0437587 & 0.0435371 & 0.0436044 & 0.0436030 & 0.0436030 & 0.0436028 \\
\hline & 0.050 & 0.0333681 & 0.0329817 & 0.0327508 & 0.0327532 & 0.0327532 & 0.0327495 \\
\hline & 0.075 & 0.0243837 & 0.0238893 & 0.0229948 & 0.0230307 & 0.0230307 & 0.0230109 \\
\hline & 0.100 & 0.0168056 & 0.0162600 & 0.0143364 & 0.0144842 & 0.0144842 & 0.0144147 \\
\hline \multirow{4}{*}{$4 p$} & 0.025 & 0.0200000 & 0.0199625 & 0.0199486 & 0.0199489 & 0.0199489 & 0.019948 \\
\hline & 0.050 & 0.0112500 & 0.0111938 & 0.0110442 & 0.0110582 & 0.0110582 & 0.0110422 \\
\hline & 0.075 & 0.0050000 & 0.0049439 & 0.0045370 & 0.0046219 & 0.0046219 & 0.0045340 \\
\hline & 0.100 & 0.0012500 & 0.0012128 & 0.0004269 & 0.0007549 & 0.000755 & 0.0004252 \\
\hline \multirow{3}{*}{$4 d$} & 0.025 & 0.02000000 & 0.0198877 & 0.0198457 & 0.0198463 & 0.0198462 & 0.0198444 \\
\hline & 0.050 & 0.0112500 & 0.0110819 & 0.0106327 & 0.0106674 & 0.0106674 & 0.0106355 \\
\hline & 0.075 & 0.0050000 & 0.0048327 & 0.0036111 & 0.0038345 & 0.0038345 & 0.0036479 \\
\hline \multirow{3}{*}{$4 f$} & 0.025 & 0.0200000 & 0.0197756 & 0.0196914 & 0.0196911 & 0.0196911 & 0.0196903 \\
\hline & 0.050 & 0.0112500 & 0.0109150 & 0.0100154 & 0.0100620 & 0.0100620 & 0.0100463 \\
\hline & 0.075 & 0.0050000 & 0.0046682 & 0.0022222 & 0.0025563 & 0.0025563 & 0.0024452 \\
\hline \multirow{2}{*}{$5 p$} & 0.025 & 0.00945312 & 0.0094325 & 0.0094017 & 0.0094036 & 0.0094036 & 0.0094011 \\
\hline & 0.050 & 0.00281250 & 0.0027900 & 0.0026067 & 0.0026490 & 0.0026490 & 0.0026047 \\
\hline
\end{tabular}

Table 10. Comparison of energy spectra $\left(-E_{n l}\right)$ for Hulthen potential as a function of the screening parameter $\frac{1}{b}$ for $2 p, 3 p, 3 d, 4 p, 4 d, 4 f, 5 p(\hbar=c=1)$ unit.

Received: 25 August 2020; Accepted: 17 November 2020

Published online: 13 January 2021

\section{References}

1. Okon, I. B., Isonguyo, C. N., Antia, A. D., Ikot, A. N. \& Popoola, O. O. Fisher and Shannon information entropies for a noncentral inversely quadratic plus exponential Mie-type potential. Commun. Theor. Phys. 72, 065104 (2020).

2. Oluwadare, O. J., Oyewumi, K. J., Akoshile, C. O. \& Babalola, O. A. Approximate analytical solutions of the relativistic equations with the Deng-Fan molecular potential including a Perkeris-type approximation to the (pseudo or) centrifugal term. Phys. Scr. 86, 035002 (2012).

3. Arda, A. \& Sever, R. Bound state solutions of the Dirac equation for the Kratzer potrential with pseudoscalar Coulomb term. Eur. Phys. J. Plus. 134, 29 (2019).

4. Bermudez, A., Martin-Degado, M. A. \& Solana, E. Mesoscopic superposition states in relativistic Landau levels. Phys. Rev. A 99, 123602 (2007).

5. Bermudez, A., Martin-Degado, M. A. \& Solana, E. Exact mapping of the $2+1$ Dirac oscillator onto the Jaynes-Cummings model: ion-trap experimental proposal. Phys. Rev. A 76, 041801 (2007).

6. Lamata, L., Martin-Degado, M. A. \& Solano, E. Relativity and Lorentz invariance of entanglement distillability. Phys. Rev. A 97, 250502 (2006).

7. Bermudez, A., Martin-Degado, M. A. \& Luis, A. Chirality quantum phase transition in the Dirac oscillator. Phys. Rev. A. 77, 063815 (2007).

8. Pakdel, F., Rajabi, A. A. \& Hamzavi, M. Scattering and bound state solutions of the Yukawa potential within the Dirac equation. Adv. High. Energy Phys. 2014, 867483 (2014).

9. Jia, C. S., Guo, P. \& Peng, X. L. Exact solution of the Dirac Eckart problem with spin and pseudospin symmetry. J. Phys. A 372, 2201 (2008).

10. Ikhdair, S. M. \& Sever, R. Exact solution of the Klein-Gordon equation for the PT-symmetric generalized Woods-Saxon potential by the Nikiforov-Uvarov method. Ann. Phys. 16, 218 (2007).

11. Ginocchio, J. N. Pseudospin as a relativistic symmetry. Phys. Rev. Lett. 78, 436 (1997).

12. Ginocchio, J. N. Relativistic symmetries in nuclei and hadrons. Phys. Rep. 414, 165-261 (2005).

13. Troltenier, D., Bahri, C. \& Draayer, J. P. Generalized pseudo-SU(3) model and pairing. Nucl. Phys. A 586, 53-72 (1995).

14. Ikhdair, S. M. \& Sever, R. Bound states of the Klein-Gordon for exponential-type potentials in D-dimensions. Appl. Math. Comput. 216, 545-555 (2010). 
15. Hamzavi, M., Eshghi, M. \& Ikhdair, S. M. Effect of Tensor interaction in the Dirac attractive radial problem under pseudospin symmetry limit. J. Math. Phys. 53, 082101 (2012).

16. Hamzavi, M., Rajabi, A. A. \& Hassanabadi, H. Exact pseudospin mass symmetry solution of the Dirac equation for spatiallydependent Coulomb potential including a Coulomb-like tensor interaction via asymptotic Iteration Method. Phys. Lett. A. 374, 4303-4307 (2010).

17. Zhang, X. C., Liu, Q. W., Jia, C. S. \& Wang, L. Z. Bound states of the Dirac equation with vector and scalar Eckart potential. Phys. Lett. A. 340, 54-64 (2005).

18. Yahya, W. A., Oyewumi, K. J., Akoshile, C. O. \& Ibrahim, T. T. Bound states of the relativistic Dirac equation with equal scalar and vector Eckart potentials using the Nikiforov-Uvarov Method. J. Vect. Relativ. 5, 1-8 (2010).

19. Berkdemir, C. Relativistic treatment of a spin-zero particles subject to a Kratzer-type potential. Am. J. Phys. 75, 81-86 (2007).

20. Guo, J. Y., Meng, J. \& Xu, F. X. Solution of the relativistic Dirac-Woods-Saxon problem. Chin. Phys. Lett. 20, 602 (2003).

21. Zhao, X. Q., Jia, C. S. \& Yang, Q. B. Bound states of relativistic particles in the generalized symmetrical double-well potential. Phys. Lett. A 337, 189-196 (2005).

22. Simsek, M. \& Grifes, E. The Klein-Gordon equation of generalized Hulthen potential in complex quantum mechanics. J. Phys. A Math. Gen. 37, 4379 (2004).

23. Chargui, Y., Trabelsi, A. \& Chetouani, L. Bound-states of the $(1+1)$-dimensional DKP equation with a pseudoscalar linear plus Coulomb-like potential. Phys. Lett. A. 374, 2907-2913 (2010).

24. Durmus, A. \& Yasuk, F. Relativistic and nonrelativistic solutions for diatomic molecules in the presence of double ring-shape potential. J. Chem. Phys. 126, 074108 (2007).

25. Zou, X., Yi, L. Z. \& Jia, C. S. Bound states of the Dirac equation with vector and scalar Eckart potentials. Phys. Lett. A 346, 54-64 (2005).

26. Agboola, D. Dirac equation with spin symmetry for the modified Poschl-Teller potential in D dimensions. Pramana J. Phys. 76, $875-885(2011)$.

27. Bayrak, O. \& Boztosun, I. The pseudospin symmetric solution of the Morse potential for any k state. J. Phys. A Math. Theor. 40, 11119-11127 (2007). https://doi.org/10.1007/s13369-001-0168-z. Eng. 137 (2011).

28. Ikhdair, S. M. \& Sever, R. Approximate eigenvalue and eigenfunction solutions for the generalized Hulthn potential with any angular momentum. J. Math. Chem. 42, 461-471 (2007).

29. Wen-Chao, Q. and Dong S. H. SUSYQM and SWKB approaches to relativistic equation with hyperbolic potential. $v_{0} \tanh \frac{r}{d}$. Phys. Scr., 72, 2-3 (2005).

30. Akbarieh, A. R. \& Motavalli, H. Exact solutions of Klein-Gordon equations for the Rosen-Morse type potential via NikiforovUvarov method. Mod. Phys. Lett. A 23, 3005-3013 (2008).

31. De Castro, A. S. Bound states of the Dirac equation for a class of effective quadratic plus inversely quadratic potentials. Ann. Phys. (N. Y.) 311, 170-181 (2004).

32. De Castro, A. S. Bounded solutions of neutral fermions with a screened Coulomb potential. Ann. Phys. (N. Y.) 320, 56-70 (2005).

33. Shabat, A. \& Zakharov, V. Exact theory of two-dimensional self-focusing and one-dimensional self-modulation of waves in nonlinear media. Sov. Phys. JETP. 34, 118-134 (1972).

34. Takhtadzhyan, L. A. \& Faddeev, L. D. Essentially nonlinear one-dimensional model of classical field theory. Theor. Math. Phys. 21, 1046-1057 (1974).

35. Dong, S. H. Factorisation Method in Quantum Mechanics (Springer, Dordrecht, 2007).

36. Infeld, I. \& Hall, T. E. The factorisation method. Rev. Mod. Phys. 23, 21-68 (1951).

37. Ciftci, H., Hall, R. \& Saad, N. Asymptotic iteration method for eigenvalue problem. J. Phy. A. 36, 11807-11816 (2003).

38. Ozer, O. \& Levai, G. Assymptotic iteration method applied to bound state problems. Rom. J. Phys. 57, 582-593 (2012).

39. Arda, A. \& Sever, R. Exact solutions of the Morse-like potential, step-up and step-down operators via Laplace transform approach. Commun. Theor. Phys. 58, 27 (2012).

40. Jia, C. S., Gao, P. \& Peng, X. L. Exact solution of the Dirac-Eckart problem with spin and pseudospin symmetry. J. Phys A Math. Gen. 39, 7737 (2006).

41. Astorga, A. C., Andez, D. J. \& Negro, J. Solutions of the Dirac equation in a magnetic field and intertwining operators. SIGMA. 8, $082(2012)$.

42. Feizi, H., Rajabi, A. A. \& Shojaei, M. R. Raising and lowering operators for the Dirac-Woods-Saxon potential in the presence of spin and pseudospin symmetry Eur. Phys. J. Plus 127, 41 (2012).

43. Cai, J., Cai, P. \& Inomata, A. Path-integral treatment of the Hulthn potential. Phys. Rev. A. 36, 4621 (1986).

44. Diaf, A., Chouchaoui, A. \& Lombard, R. J. Feynman integral treatment of the Bargmann potential. Ann. Phys. 317, 354 (2005).

45. Diaf, A. \& Chouchaoui, A. l-states of the Manning-Rosen potential with an improved approximate scheme and Feynman path integral formalism. Phys. Scr. 84, 015004 (2011).

46. Shojaei, M. R., Rajabi, A. A., Farrokh, M. \& Zoghi-Foumani, N. Energy levels of spin-1/2 particles with Yukawa interaction. Int. J. Mod. Phys. 5, 773-780 (2014).

47. Xu, Y., He, S. \& Jia, C.-S. Approximate analytical solutions of the Klein-Gordon equation with Poschl-Teller potential including centrifugal term. Phys. Scr. 81, 045001 (2010).

48. Oluwadare, O. J., Oyewumi, K. J. \& Babalola, O. A. Exact S-waves solution of the Klein-Gordon equation with the Deng-Fan molecular potential using Nikiforov-Uvarov method. Afr. Rev. Phys. 7, 0016 (2012).

49. Cheng, Y.-F. \& Dai, T.-Q. Exact solutions of the Klein-Gordon equation with a ring-shaped modified Kratzer potential. Chin. J. Phys. 45, 480-487 (2007).

50. Zhang, X. C., Liu, Q. W., Jia, C. S. \& Wang, S. Z. Bound states of the Dirac equation with vector and scalar Scarf-type potentials. Phys. Lett. A. 340, 59 (2005).

51. Guo, J. Y. \& Sheng, Z. Q. Solution of the Dirac equation for the Woods-Saxon potential with spin and pseudospin symmetry. Phys. Lett. A. 338, 90 (2005).

52. Scarf, F. New soluble energy band problem. Phys. Rev. 112, 1137-1140 (1958).

53. Chen, C. Y. Exact solutions of the Dirac equation with scalar and vector Hartmann potentials. Phys. Lett. A. 339, $283-287$ (2005).

54. Falaye, B. J. Any l-state solutions of the Eckart potential via asymptotic iteration method. Cent. Euro. J. Phys. 10, 960-965 (2012).

55. Falaye, B. J., Ikhdair, S. M. \& Hamzavi, M. Formula method for bound state problems. Few-Body Syst. 56, 63-78 (2015).

56. Shojaei, M. R. \& Mousavi, M. Calculation energy levels and charge radius for odd 41-49 Ca isotopes by using the analytical approach. Adv. High Energy. Phys. 2016, 12 (2016).

57. Dutra, A. D. \& Hott, M. Dirac equation exact solutions for generalized asymmetrical Hartmann potentials. Phys. Lett. A. 356, 215 (2006).

58. Okorie, U. S., Ibekwe, E. E., Onyeaju, M. C. \& Ikot, A. N. Solutions of the Dirac and Schrodinger equations with shifted Tietz-Wei potential. Eur. Phys. J. Plus 133, 433 (2018).

59. Onyeaju, M. C. et al. Approximate bound-states solution of the Dirac equation with some thermodynamic properties for the deformed Hylleraas plus deformed Woods-Saxon potential. Eur. Phys. J. Plus 132, 302 (2017).

60. Shui, Z. W. \& Jia, C. S. Relativistic rotation-vibrational energies for the 107Ag 109Ag isotope. Eur. Phys. J. Plus 132, 292 (2017).

61. Ortakaya, S., Hassanabadi, H. \& Yazarloo, B. H. Bound state solutions of the Dirac equation with Deng-Fan potential including Coulomb and tensor interaction. Chin. Phys. B 23, 3 (2014). 
62. Ikot, A. N. Solutions of Dirac equation for generalized hyperbolical potential including Coulomb-like tensor potential with spin symmetry. Few-Body Syst. 53, 549-555 (2012).

63. Ikot, A. N., Maghsoodi, E., Antia, A. D., Zarrinkamar, S. \& Hassanabadi, H. Approximate k-state solutions to the Dirac Mobius square-Yukawa and Mobius square-quasi Yukawa problems under pseudospin and spin symmetry limits with Coulomb-like tensor interaction. Can. J. Phys. 91, 560-575 (2013).

64. Dong, S. H., Qiang, W. C., Sun, G. H. \& Bezerra, V. B. Analytical approximations to the l-wave solutions of the Schrodinger equation with the Eckart potential. J. Phys. A Math. Theor. 40, 10535 (2007).

65. Jia, C. S. \& Jia, Y. Relativistic rotation-vibrational energies for the Cs2 molecule. Eur. Phys. J. D 71, 3 (2017).

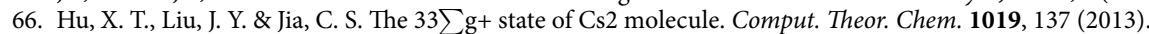

67. Ikhdair, S. M. Rotational and vibrational diatomic molecule in the Klein-Gordon equation with hyperbolic scalar and vector potential. Int J. Mod. Phys. C 20, 1563-1582 (2009).

68. Ikot, A. N. et al. Thermodynamic properties of Aharanov-Bohm (AB) and magnetic fields with screened Kratzer potential. Eur. Phys. J. D. 74, 159 (2020).

69. Dong, S., Sun, G.-H., Dong, S.-H. \& Draayer, J. P. Quantum information entropies for a squared tangent potential well. Phys. Lett. A. 378, 124-130 (2014).

70. Okon, I. B., Antia, A. D., Akankpo, A. O. \& Essien, I. E. Eigen-solutions to Schrodinger equation with trigonometric inversely quadratic plus Coulombic hyperbolic potential. Phys. Sci. Int. J. 24, 61-75 (2020).

71. Onate, C. A. Bound state solutions of the Schrodinger equation with second Poschl-Teller like potential model and the vibrational partition function, mean energy and mean free energy. Chin. J. Phys. 54, 138-165 (2016).

72. Okorie, U. S., Ikot, A. N., Chukwuuocha, E. O. \& Rampho, G. J. Thermodynamic properties of improved exponential-type potential (IDEP) for some diatomic molecules. Results Phys. 17, 103078 (2020).

73. Jia, C.-S., Liu, J.-Y. \& Wang, P.-Q. A new approximation scheme for the centrifugal term and the Hulthenn potential. Phys. Lett. A 372, 4779-4782 (2008).

74. Ikhdair, S. M. An improved approximation scheme for the centrifugal term and the Hulthen potential. Eur Phy. J. A 39, 307-314 (2009).

75. Bayrak, O., Kocak, G. \& Boztosun, I. Any l-state solutions of the Hulthen potential by the asymptotic iteration method. J. Phys A Math. Gen. 39, 11521-11529 (2006).

76. Varshni, Y. P. Eigenenergies and oscillator strengths for the Hulthen potential. Phys. Rev. A. 41, 4682 (1990).

77. Stanek, J. Approximate analytical solutions for arbitrary l-state of the Hulthen potential with an improved approximation of the centrifugal term. Cent. Eur. J. Chem. 9, 737-742 (2011).

78. Okon, I. B., Popoola, O. O. \& Isonguyo, C. N. Approximate solutions of Schrodinger equation with some diatomic molecular interactions using Nikiforov-Uvarov method. Adv. High Energy Phys. 2017, 9671816 (2017).

79. Tezcan, C. \& Sever, R. A. General approach for the exact solution of the Schrodinger equation. Int. J. Theor. Phys. 48, 337-350 (2009).

\section{Acknowledgements}

The authors acknowledge the contribution of ICTP for communicating some of the research materials as well the reviewers for their comments and positive suggestions which have improved the present article.

\section{Author contributions}

I.B.O., E.O. and A.D.A. designed and wrote the main manuscript. C.O., L.E.A. and O.E.O. did the computations, prepared the figures and the tables. All authors reviewed the manuscript.

\section{Competing interests}

The authors declare no competing interests.

\section{Additional information}

Correspondence and requests for materials should be addressed to I.B.O.

Reprints and permissions information is available at www.nature.com/reprints.

Publisher's note Springer Nature remains neutral with regard to jurisdictional claims in published maps and institutional affiliations.

(c) (i) Open Access This article is licensed under a Creative Commons Attribution 4.0 International cc) License, which permits use, sharing, adaptation, distribution and reproduction in any medium or format, as long as you give appropriate credit to the original author(s) and the source, provide a link to the Creative Commons licence, and indicate if changes were made. The images or other third party material in this article are included in the article's Creative Commons licence, unless indicated otherwise in a credit line to the material. If material is not included in the article's Creative Commons licence and your intended use is not permitted by statutory regulation or exceeds the permitted use, you will need to obtain permission directly from the copyright holder. To view a copy of this licence, visit http://creativecommons.org/licenses/by/4.0/.

(c) The Author(s) 2021 